\title{
Steady flow past a sphere in an aligned magnetic field at high Reynolds numbers
}

\author{
Harish Kumar $^{1^{*}}$, R. Rajathy ${ }^{2}$ \\ ${ }^{1 *}$ Department of Physics, Pondicherry Engineering College, Pondicherry-605 014, INDIA \\ ${ }^{2}$ Department of Electrical and Electronics Engineering, Pondicherry Engineering College, Pondicherry-605 014, INDIA \\ "Corresponding author: e-mail: harishkumarholla@pec.edu, Tel: +91-9443052957, Fax: +91-413-2655101
}

\begin{abstract}
The flow of steady, incompressible, viscous, electrically conducting fluid past a sphere in the presence of an uniform magnetic field parallel to the undisturbed flow is investigated using the finite difference method. The multigrid method with defect correction technique is used to achieve the second order accurate solution. The Hartmann number, $M$ is used as the perturbation parameter. It is found that the increase of magnetic field decreases the wake length and increases the drag coefficient. The graphs of streamlines, vorticity lines, drag coefficient, surface pressure and surface vorticity are presented and discussed.
\end{abstract}

Keywords: Navier-Stokes equations, MHD, Hartmann number, Multigrid method, Defect correction.

DOI: http://dx.doi.org/10.4314/ijest.v6i1.1

\section{Introduction}

The flow of viscous, incompressible and electrically conducting fluid past a sphere in the presence of a parallel magnetic field was first investigated by Chester (1957). Subsequently it was discussed by Ludford (1960) and Cabannes (1970). The work of Yosinobu et al. (1959) on axisymmetric flow past a cylinder in aligned and transverse magnetic field for small Hartmann number was generalised by Savage (1972) for arbitrary direction of the magnetic field. Swarup et al. (1977) studied the same problem with aligned magnetic field in terms of Hartmann number. All these studies concentrated on the flow at low and intermediate values of Reynolds number and the main observation was that the magnetic field tends to stabilize the flow. Turkyilmazoglu (2011) studied the boundary layer flow of a steady, laminar, incompressible, viscous and electrically conducting fluid arising due to a rotating sphere, subjected to a uniform suction and injection through the surface, in the presence of a uniform radial magnetic field. The stability of the boundary layer flow due to rotating spheres, disks and cones have been studied theoretically and experimentally by Garret et al. (2002, 2004, 2007, 2009), Turkyilmazoglu (2006) and Kohama et al. (1983). They found that at high Reynolds number the boundary layer becomes unstable.

Viscous flow past a sphere becomes unstable around Reynolds number $R e=130$ and past a circular cylinder around $R e=40$. We are interested in the structure of the steady (but unstable) solutions above these Reynolds numbers. At high Reynolds numbers, the conventional analytic methods are not suitable due to the highly non-linear nature of Navier-Stokes equations. As a consequence, numerical methods are gaining interest among the researchers. In the high Reynolds number regime, the only existing results for the steady viscous flow past a sphere is due to Le Clair et al. (1970) (for Re upto 400) and Fornberg (1988) (up to $R e=5000$ ). But both Le Clair et al. and Fornberg did not consider the application of magnetic field and its effects. It would be interesting to see the effect of aligned magnetic field on the behaviour of the solutions at higher $R e$, about which, unfortunately, no literature exists. In the two dimensional flow of a liquid under the influence of an applied magnetic field, it is proved that if the field is strong enough and has the appropriate orientation, the separation of a viscous boundary layer can be prevented even up to the rear stagnation point. Leibovich (1967) suggested that the separation at rear stagnation point of a circular 
cylinder could be suppressed by a sufficiently large magnetic field perpendicular to the surface of the cylinder. The suppression of the separation bubble at the rear stagnation point is also observed for cylinder by Raghava Rao et al. (2000), who approximated the convective terms by first order upwind difference scheme. They observed that the drag coefficient increases with the magnetic field. However, their results are not second order accurate. In this paper, we discuss the flow of a conducting fluid past a sphere at high Reynolds numbers $(R e=100-1000)$ and for the intermediate values of Hartmann number $M$ using the multigrid (MG) method. Compared to the Newton's method used by Fornberg, the MG method is less demanding on the computational resources. We were able to get solutions for values of $R e$ upto 1000 using a personal computer with core2duo processor (2.2GHz) and a RAM of 2GiB. However, the multigrid method gives only first order accurate solution (due to the upwind discretization). The theoretical and experimental data presented in the literature (Clift et al., 1978) suggests that second order accuracy is at least desirable. We employed the defect correction technique (Juncu, 1990; Juncu et al., 1999) to obtain the second order accurate solution and it seems to be most adequate for the job.

\section{Formulation of the Problem}

The equations governing the steady flow of an incompressible fluid (with finite electrical conductivity $\sigma$ ) past a sphere (of radius $a$ ) with uniform free-stream velocity $\mathbf{U}_{\infty}$ and an uniformly applied magnetic field $\mathbf{H}_{\infty}$ at large distances are, in nondimensional form,

$$
\begin{gathered}
\frac{R e}{2}(\mathbf{q} \cdot \nabla) \mathbf{q}=-\nabla p+\nabla^{2} \mathbf{q}+\frac{M^{2}}{R_{m}}[(\nabla \times \mathbf{H}) \times \mathbf{H}] \\
\mathbf{j}=\nabla \times \mathbf{H}=\frac{R_{m}}{2}[\mathbf{E}+\mathbf{q} \times \mathbf{H}] \\
\nabla \cdot \mathbf{q}=0 \\
\nabla \cdot \mathbf{H}=0 \\
\nabla \times \mathbf{E}=0
\end{gathered}
$$

where $p$ is the pressure, $\mathbf{q}$ is the fluid velocity, $\mathbf{H}$ is the magnetic field, $\mathbf{E}$ is the electric field, and $\mathbf{j}$ is the current density. The Reynolds number is $\operatorname{Re}=2 \rho U_{\infty} a / \eta$ and $M=\mu H_{\infty} a(\sigma / \eta)^{1 / 2}$ is the Hartmann number. The magnetic Reynolds number is given by $R_{m}=U_{\infty} a \mu \sigma$. The viscosity, density and magnetic permeability of the fluid are $\eta, \rho$ and $\mu$ respectively. In order to satisfy equation (3), the dimensionless stream function $\psi(r, \theta)$ is introduced such that

$$
u=\frac{1}{r^{2} \sin \theta} \frac{\partial \psi}{\partial \theta}, v=-\frac{1}{r \sin \theta} \frac{\partial \psi}{\partial r}
$$

where $u$ and $v$ are the dimensionless radial and transverse components of fluid velocity. Spherical polar co-ordinates $(r, \theta, \varphi)$ are used as they are the most suitable in dealing with a spherical boundary. The co-ordinate system is set up in such a way that the flow is symmetric about $\theta=0^{\circ}$ and $\theta=180^{\circ}$. Since the flow is two dimensional, equations (2) and (5) give

$$
\mathbf{E}=(0,0,0)
$$

The problem can be simplified by assuming the magnetic Reynolds number to be small and the magnetic field to be weak enough so that its effect can be treated as a small perturbation of the potential flow with no magnetic field. Hence we can use the low- $R_{m}$ approximation and ignore equation (2) as well as replace the magnetic field in all MHD equations by

$$
\mathbf{H}=(-\cos \theta, \sin \theta, 0) \text {, }
$$

which will eliminate several non linear terms of unknown quantities in the governing equations. Pressure can be eliminated from equation (1) to get

$$
\frac{R e}{2}\{\nabla \times(\omega \times \mathbf{q})\}=\nabla^{2} \omega+\frac{M^{2}}{R_{m}}[\nabla \times\{(\nabla \times \mathbf{H}) \times \mathbf{H}\}]
$$

where

$$
\omega=\nabla \times \mathbf{q}
$$


is the vorticity. Substitution of equation (2) in equation (9) gives

$$
\nabla^{2} \omega=\frac{R e}{2}[\nabla \times(\omega \times \mathbf{q})]-\frac{M^{2}}{2}[\nabla \times\{(\mathbf{q} \times \mathbf{H}) \times \mathbf{H}\}]
$$

Using equations (6) and (8) along with the transformation $r=e^{\xi}$, equations (10) and (11) can be written as

$$
\frac{\partial^{2} \psi}{\partial \xi^{2}}-\frac{\partial \psi}{\partial \xi}+\frac{\partial^{2} \psi}{\partial \theta^{2}}-\cot \theta \frac{\partial \psi}{\partial \theta}+\sin \theta e^{3 \xi} \omega=0
$$

and

$$
\begin{gathered}
\frac{\partial^{2} \omega}{\partial \xi^{2}}+\frac{\partial \omega}{\partial \xi}+\cot \theta \frac{\partial \omega}{\partial \theta}+\frac{\partial^{2} \omega}{\partial \theta^{2}}-\frac{\omega}{\sin ^{2} \theta}=\frac{R e}{2 \sin \theta} e^{-\xi}\left[\frac{\partial \psi}{\partial \theta} \frac{\partial \omega}{\partial \xi}-\frac{\partial \psi}{\partial \xi} \frac{\partial \omega}{\partial \theta}-\omega \frac{\partial \psi}{\partial \theta}+\omega \cot \theta \frac{\partial \psi}{\partial \xi}\right] \\
-\frac{M^{2}}{2 \sin \theta} e^{-\xi}\left[\sin 2 \theta \frac{\partial \psi}{\partial \theta}-\sin 2 \theta \frac{\partial^{2} \psi}{\partial \xi \partial \theta}+\sin ^{2} \theta \frac{\partial^{2} \psi}{\partial \theta^{2}}+\cos ^{2} \theta \frac{\partial^{2} \psi}{\partial \xi^{2}}-\cos 2 \theta \frac{\partial \psi}{\partial \xi}\right]
\end{gathered}
$$

in the vorticity-stream function form. Equations (12) and (13) must now be solved subject to the following boundary conditions: (1) On the surface of the sphere; $(r=1), \xi=0$,

$$
\begin{gathered}
\psi=\frac{\partial \psi}{\partial \xi}=0 \\
\omega=-\frac{1}{\sin \theta} \frac{\partial^{2} \psi}{\partial \xi^{2}}
\end{gathered}
$$

(2) At large distances from the sphere; $(r \rightarrow \infty), \xi \rightarrow \infty$,

$$
\begin{gathered}
\psi \sim \frac{1}{2} e^{2 \xi} \sin ^{2} \theta \\
\omega \rightarrow 0
\end{gathered}
$$

(3) Along the axis of symmetry $\left(\theta=0^{\circ}, \theta=180^{\circ}\right), \psi=0$ and $\omega=0$.

\section{Numerical method}

The coupled nonlinear partial differential equations are solved by first applying finite difference method and the resulting algebraic equations are solved by using the multigrid method. Here, a recursive multigrid procedure is employed in which the smoother is a point Gauss Seidel iteration and the usual coarse grid correction is applied as follows (Wesseling 1991).

Let there be a sequence of computational grids $G^{1}, G^{2}, \ldots, G^{l}$ with $G^{k}$ finer than $G^{k-1}$. Let $U^{k} \rightarrow \mathbf{R}$ be the space of grid functions on $G^{k}$, let $P^{k}: U^{k-1} \rightarrow U^{k}$ be a prolongation operator and let $R^{k}: U^{k+1} \rightarrow U^{k}$ be a restriction operator. Suppose we have a non linear (system of) partial differential equation(s), discretized on $G^{1}, G^{2}, \ldots, G^{l}$. On $G^{k}$, the algebraic problem to be solved is given by

$$
A^{k}\left(u^{k}\right)=f^{k}
$$

where, $A^{k}$ is the matrix obtained by suitable discretization. If $\hat{u}$ is the approximation to exact solution $u$, then, $(\hat{u}-u)$ represents the error $e$. Then, we have

$$
A e=-r=A \hat{u}-f
$$

where, $r$ is called the residue. The coarse grid approximation $\bar{u}$ of $-e$ satisfies

$$
\bar{A} \bar{u}=R r
$$

where, $\bar{A}$ is the operator obtained by discretizing the original problem on a coarser grid and $R$ is the restriction operator. If the grid under consideration is coarsest, then the above equation should be solved exactly. The coarse grid correction to be added to $\hat{u}$ is $P \bar{u}$ (where $P$ is the prolongation operator) given by 


$$
\hat{u}=\hat{u}+P \bar{u}
$$

This represents one multigrid cycle. Solving on $G^{l-1}$ by $\gamma$ multigrid iterations results in the following recursive algorithm: procedure $\operatorname{MG}(k, u, f)$

begin if $k=1$ then solve $A^{1}\left(u^{1}\right)=f^{1}$ else

begin $S_{1}(k, u, f)$

$$
\begin{aligned}
& \text { Choose } \tilde{u}^{k-1} \in U^{k-1} \\
& \tilde{f}^{k-1}=A^{k-1}\left(\tilde{u}^{k-1}\right) \\
& f^{k-1}=\tilde{f}^{k-1}+R^{k-1}\left(f^{k}-A^{k}\left(u^{k}\right)\right) \\
& \text { for } i:=1 \text { step } 1 \text { until } \gamma \text { do } \operatorname{MG}(k-1, u, f) \\
& u^{k}=u^{k}+P^{k}\left(u^{k-1}-\tilde{u}^{k-1}\right) \\
& S_{2}(k, u, f)
\end{aligned}
$$

end

end MG

where, $S$ denotes a smoother involving a small number of point Gauss Seidel iterations.

The initial solution is taken as $\psi=0$ and $\omega=0$ at all inner grid points except for $\psi$ at $\xi=\infty$ where the boundary condition holds. In finding the solution for higher values of $R e$ and $M$, the solution obtained for lower values of $R e$ and $M$ are used as starting solution. Among the two variables, $\omega$ and $\psi$, we first solved for $\omega$ and then for $\psi$. Convergence is said to have been achieved when the difference between two successive iterations $m$ and $m+1$, at all interior grid points, is less than $10^{-5}$, i.e.,

$$
\left|\psi^{m+1}-\psi^{m}\right|<10^{-5}
$$

and

$$
\left|\omega^{m+1}-\omega^{m}\right|<10^{-5}
$$

The restriction operator $R_{k}^{k-1}$ transfers a fine grid function $U^{k}$ to a coarse grid function $U^{k-1}$. On the other hand the prolongation operator, denoted as $P_{k-1}^{k}$, transfers a coarse grid function $U^{k-1}$ to a fine grid function $U^{k}$. For the restriction operator, the simplest form is 'injection' where by the values of a function in the coarse grid are taken to be exactly the values at the corresponding points of the next fine grid i.e.,

$$
\left(R_{k}^{k-1} u^{k}\right)_{i+1, j+1}=u_{2 i+1,2 j+1}^{k} \text {. }
$$

We used the above injection operator throughout this study. For the prolongation operator the simplest form is derived using linear interpolation. Prolongation by linear interpolation introduces no ambiguity when the interpolated value is desired at the mid points of the boundaries of a mesh cell. The following 9-point prolongation operator defined by Wesseling (1980) is used for the present study

$$
\begin{gathered}
\left(P_{k-1}^{k} u^{k-1}\right)_{2 i+1,2 j+1}=u_{i+1, j+1}^{k-1} \\
\left(P_{k-1}^{k} u^{k-1}\right)_{2 i+2,2 j+1}=\frac{1}{2}\left(u_{i+1, j+1}^{k-1}+u_{i+2, j+1}^{k-1}\right) \\
\left(P_{k-1}^{k} u^{k-1}\right)_{2 i+1,2 j+2}=\frac{1}{2}\left(u_{i+1, j+1}^{k-1}+u_{i+1, j+2}^{k-1}\right) \\
\left(P_{k-1}^{k} u^{k-1}\right)_{2 i+2,2 j+2}=\frac{1}{4}\left(u_{i+1, j+1}^{k-1}+u_{i+2, j+1}^{k-1}+u_{i+1, j+2}^{k-1}+u_{i+2, j+2}^{k-1}\right) .
\end{gathered}
$$

The solution obtained by the above method is not second order accurate as we have approximated all terms by second order central difference method except convective terms which are approximated by first order upwind difference scheme to ensure diagonal dominance. In order to achieve second order accurate solution, the defect correction method is employed as follows. With B as the operator obtained, for example, by first order upwind discretization and $\mathbf{A}$ is that obtained by second order accurate discretization, the defect correction algorithm (Juncu (1990), Juncu et al. (1999)) works as given below. 
At the start of defect correction, $\bar{y}$ is a solution that is not second order accurate, and at the end of defect correction, $\bar{y}$ is second order accurate.

begin Solve $\mathbf{B} \bar{y}=b$

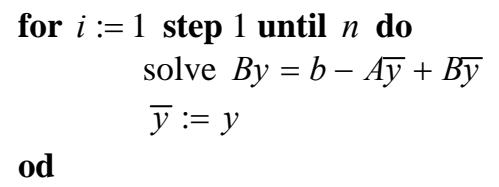

end

Usually, in practice, it is sufficient to take $n=1$ or 2 .

\section{Results and discussions}

The multigrid method with coarse grid correction is applied to solve the resulting algebraic equations which enhances the convergence rate. The computational grid extended up to 60 times the radius of the sphere. Five grids are taken with $512 \times 512$ as finest grid and $32 \times 32,64 \times 64,128 \times 128$ and $256 \times 256$ as coarser grids. Results obtained from the finest grid $512 \times 512$ of the above multigrid procedure are presented for the range of the Reynolds numbers 100 to 1000 and for the intermediate values of Hartmann number. We found that first order upwind difference scheme approximation to the non-linear convective terms ensures diagonal dominance even at high Reynolds numbers (upto $R e=1000$ in this study). So we could apply Point Gauss-Seidel method to solve the algebraic system throughout this study. Defect correction (DC) technique is then employed to achieve second order accuracy.

As a check for our method, we solved the problem for some low Reynolds numbers. We observed the separation at the rear stagnation point for all $R e \geq 25$ in $M=0$ case. The length of the wake is found to increase with $R e$ as observed by some researchers [LeClair et al. (1970), Dennis et al. (1971), Fornberg (1988)] which gives the confidence that the method is correct. We observed that as the magnetic field is increased, the $\mathbf{j} \times \mathbf{H}$ forces dominate and produce a convective rate in a direction opposite to the flow resulting in the decrease of wake length (Figs. 1-8) and separation angle (Fig. 9) for all $R e$ values under consideration. A similar phenomenon was observed by Raghava Rao et al. $(1993,1995)$ in the case of the translation of a sphere in a rotating viscous fluid and MHD flow past a circular cylinder (2000). At $R e=1000$ and $M=0$, we observed a second separation bubble between $\theta=48.77^{\circ}$ and $\theta=55.78^{\circ}$ due to the weakening of tangential stresses, which is also observed by Fornberg (1988). This indicates that the flow is becoming turbulent. But as $M$ increases, this bubble is suppressed and eventually disappears (Figs. 7-8).

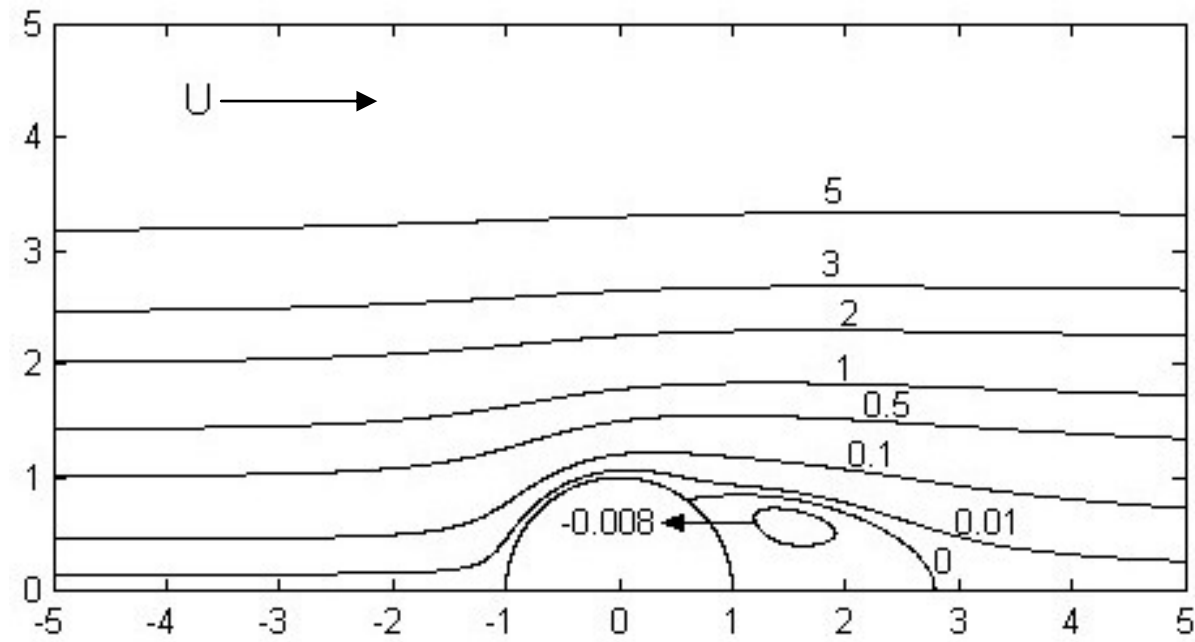

Figure 1. Streamlines for $R e=100$ and $M=0$. 


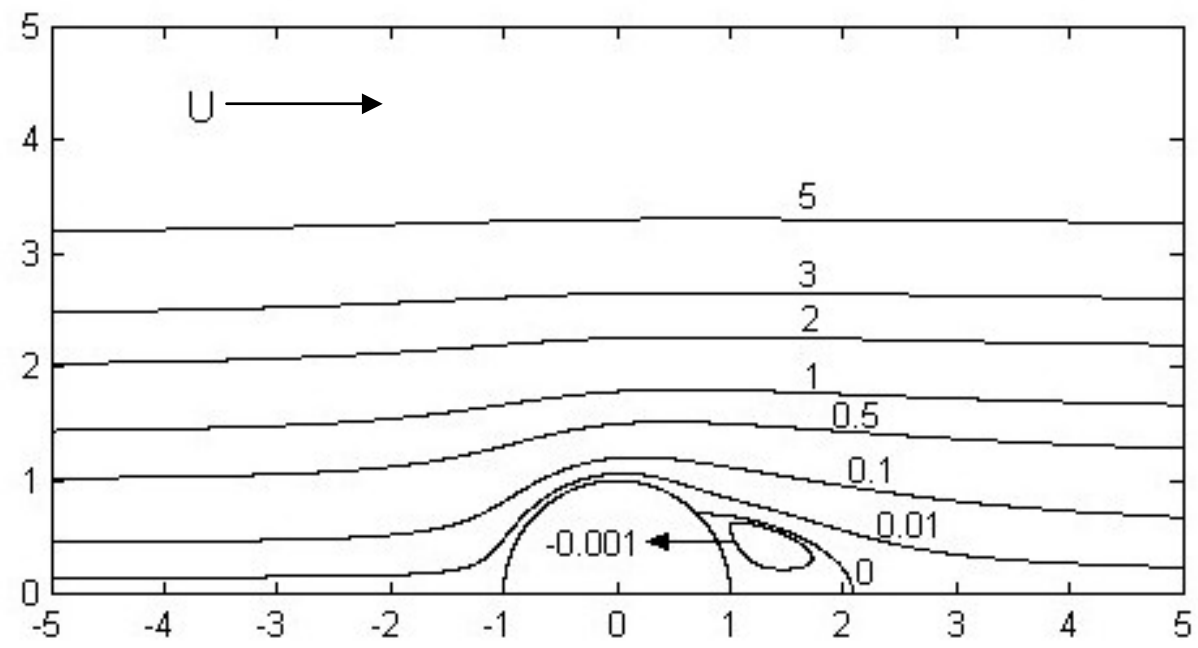

Figure 2. Streamlines for $R e=100$ and $M=10$.

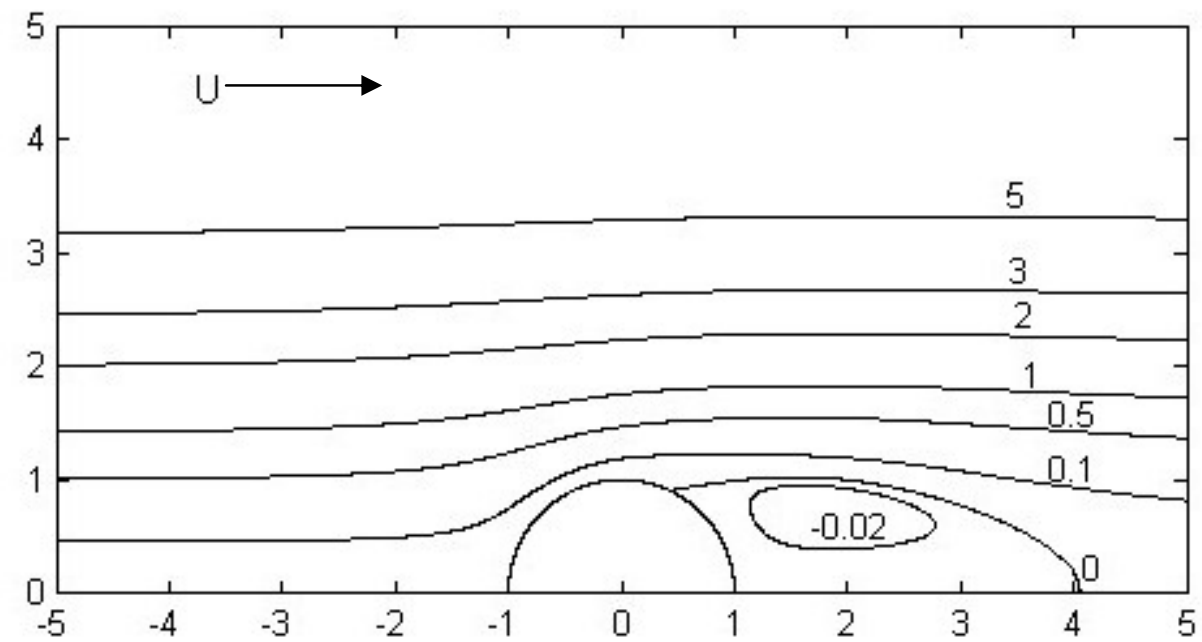

Figure 3. Streamlines for $R e=200$ and $M=0$.

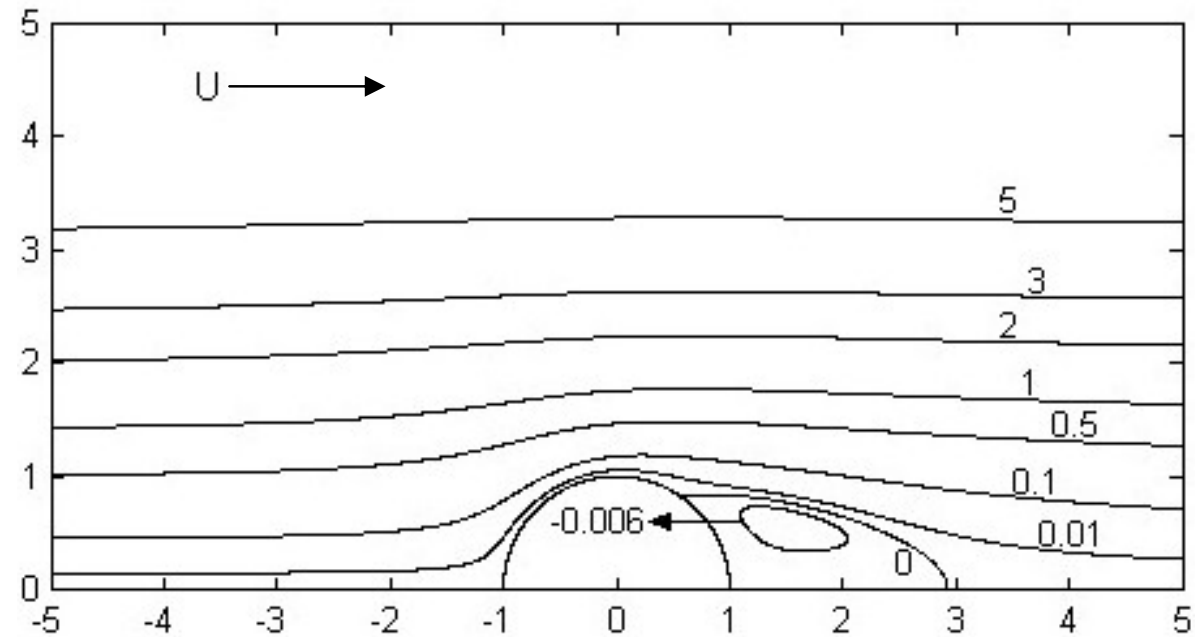

Figure 4. Streamlines for $R e=200$ and $M=14$. 


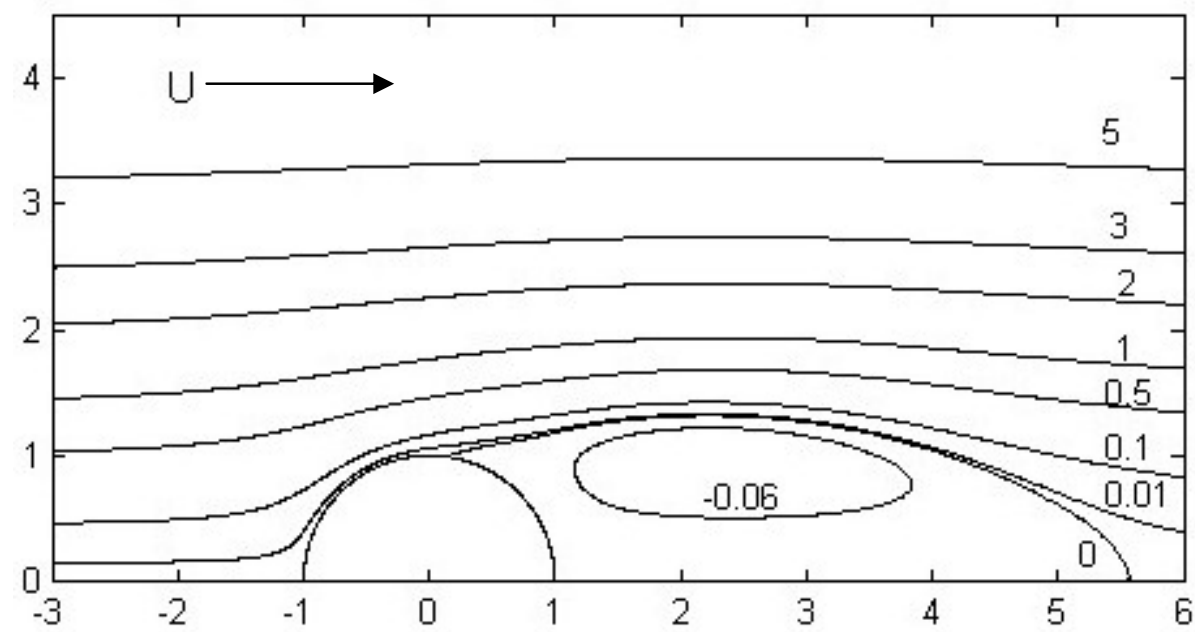

Figure 5. Streamlines for $R e=500$ and $M=0$.

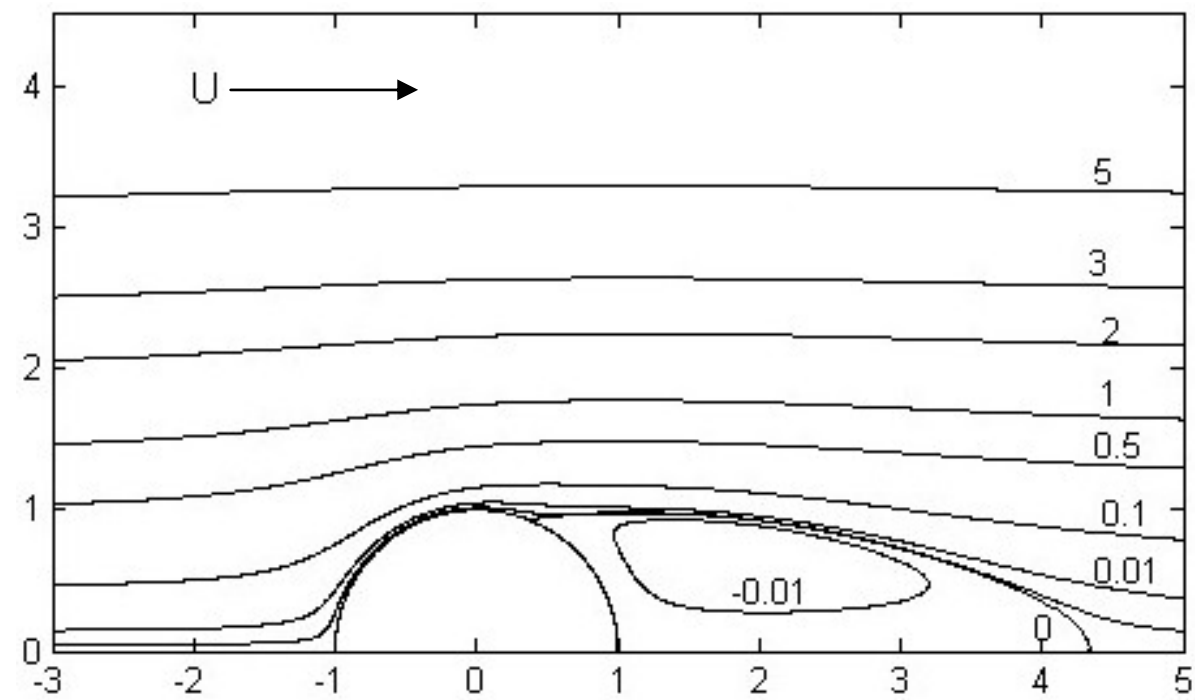

Figure 6. Streamlines for $R e=500$ and $M=20$.

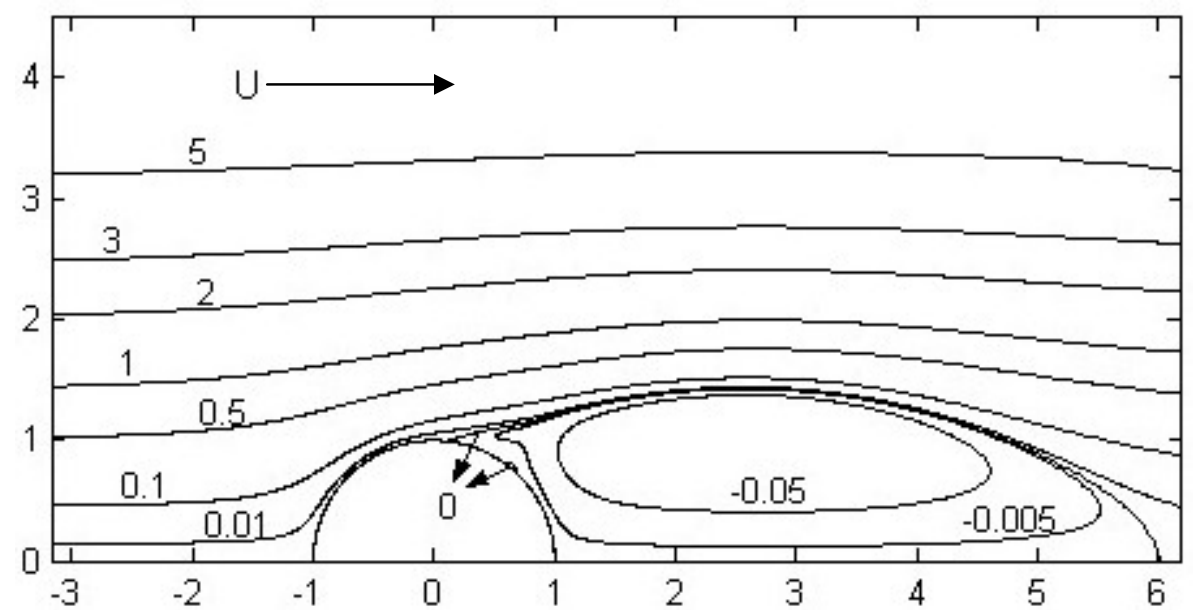

Figure 7. Streamlines for $R e=1000$ and $M=0$. 


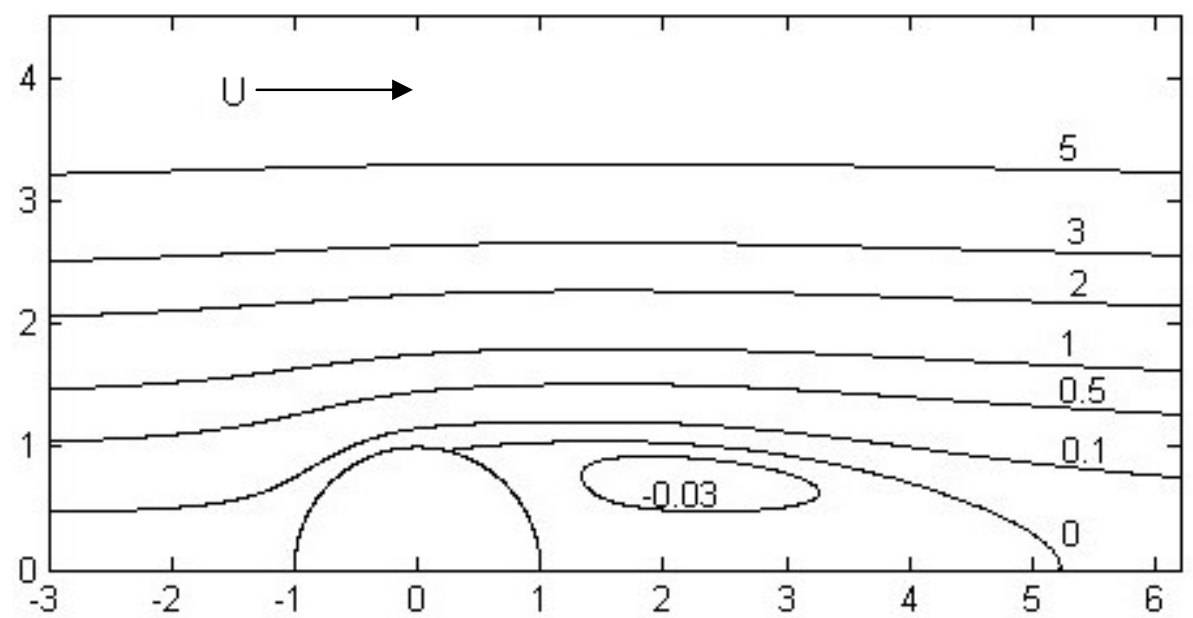

Figure 8. Streamlines for $R e=1000$ and $M=30$.

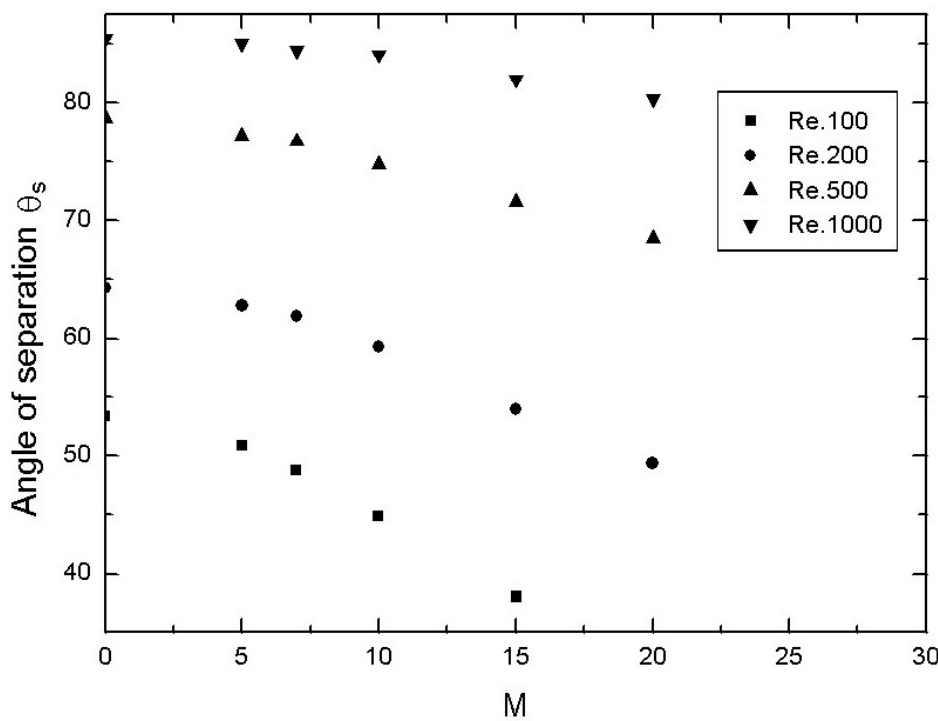

Figure 9. Hartmann number versus angle of separation

At the higher Reynold's numbers, we found that the vorticity distribution in the wake bubble resembles that of a Hill's spherical vortex as observed by Fornberg (1988) and this resemblance disappears for increased values of $M$ due to opposite convection (Figs. 10-12).

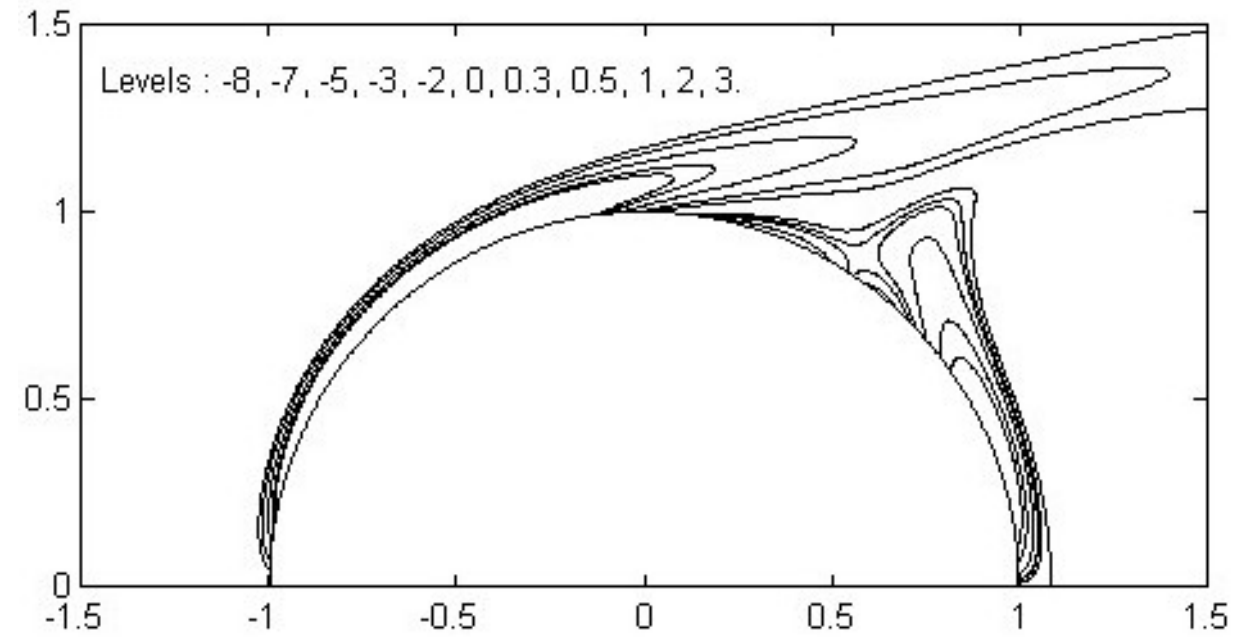

Figure 10. Vorticity distribution in the wake bubble for $R e=1000$ and $M=0$. 


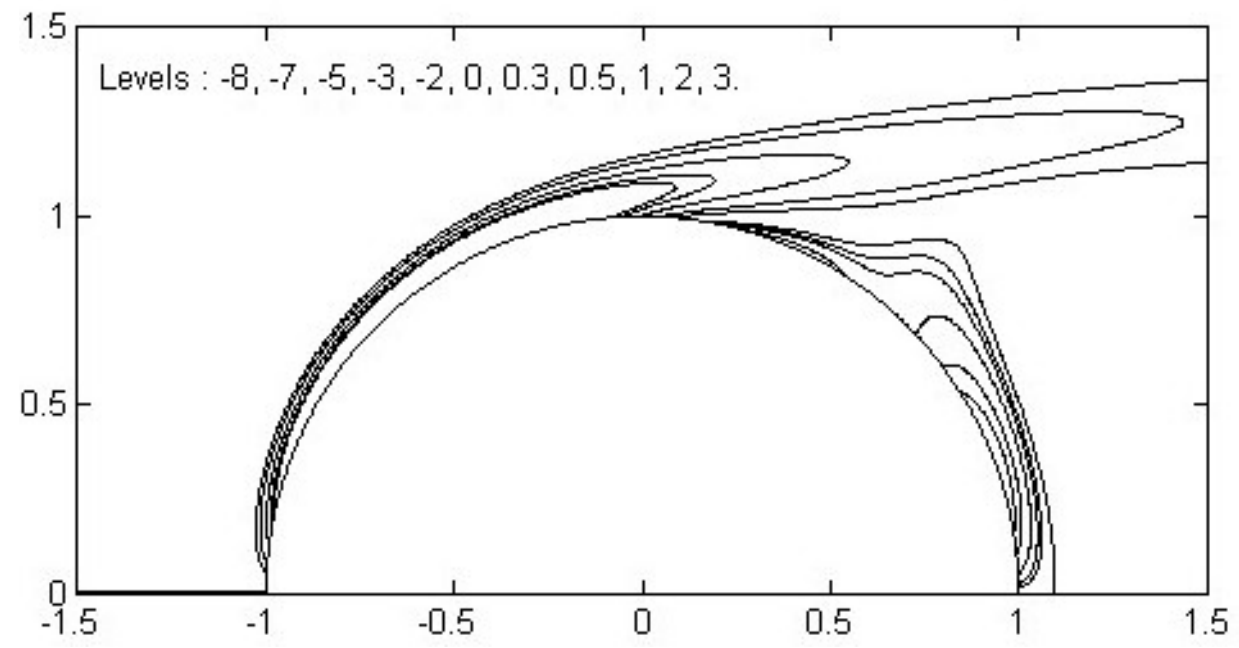

Figure 11. Vorticity distribution in the wake bubble for $R e=1000$ and $M=20$.

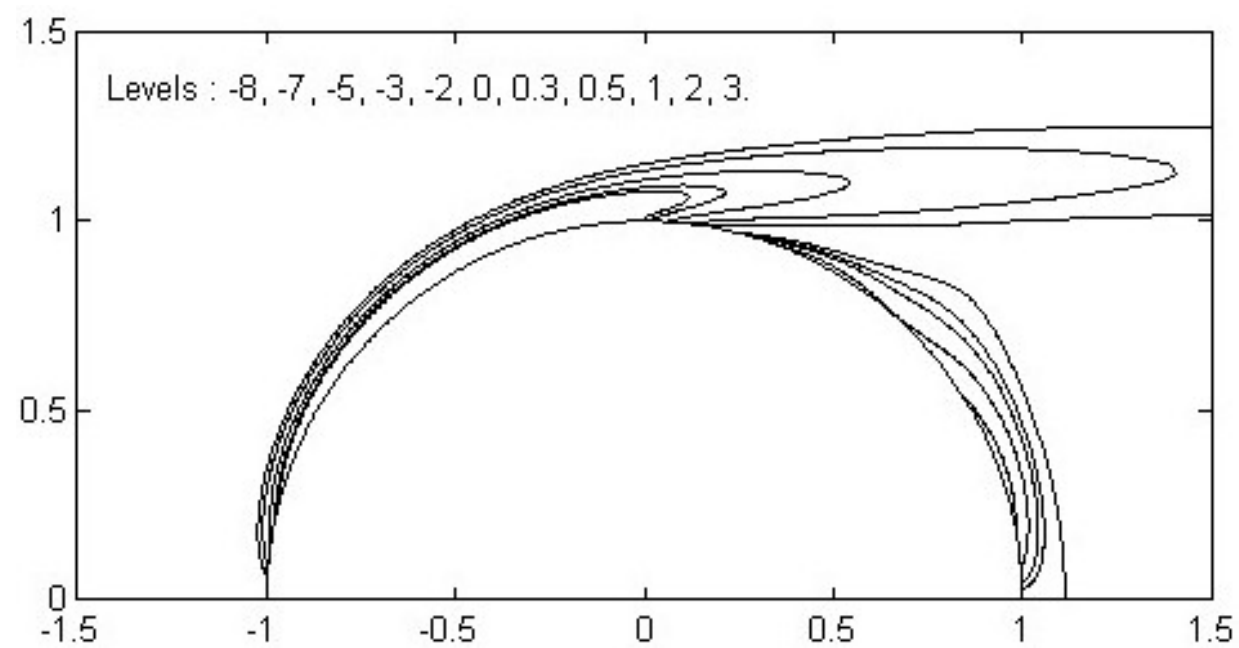

Figure 12. Vorticity distribution in the wake bubble for $R e=1000$ and $M=30$.

Since the magnetic forces are proportional to and resist the flow of fluid in any other direction than that of the unperturbed magnetic field, near the sphere, they produce a change in the pattern of the vorticity lines. The length of standing vortex is reduced slightly and the strength of the disturbance in front of the sphere is increased with increasing magnetic field (Figs. 13-20).

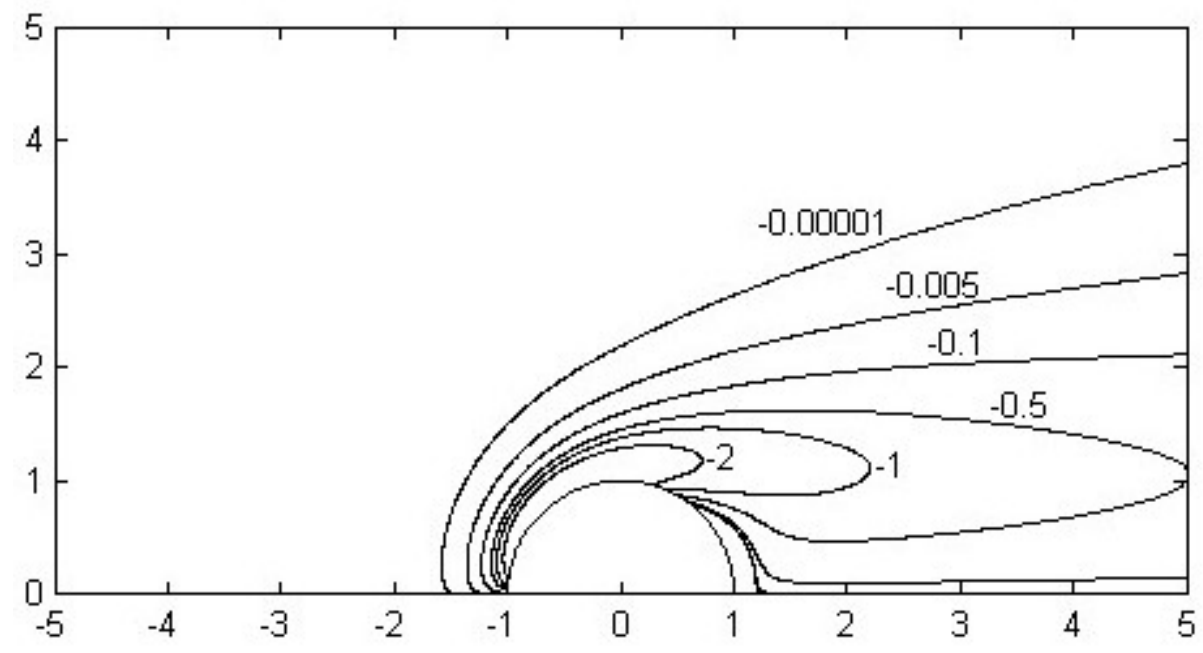

Figure 13. Vorticity lines for $R e=100$ and $M=0$. 


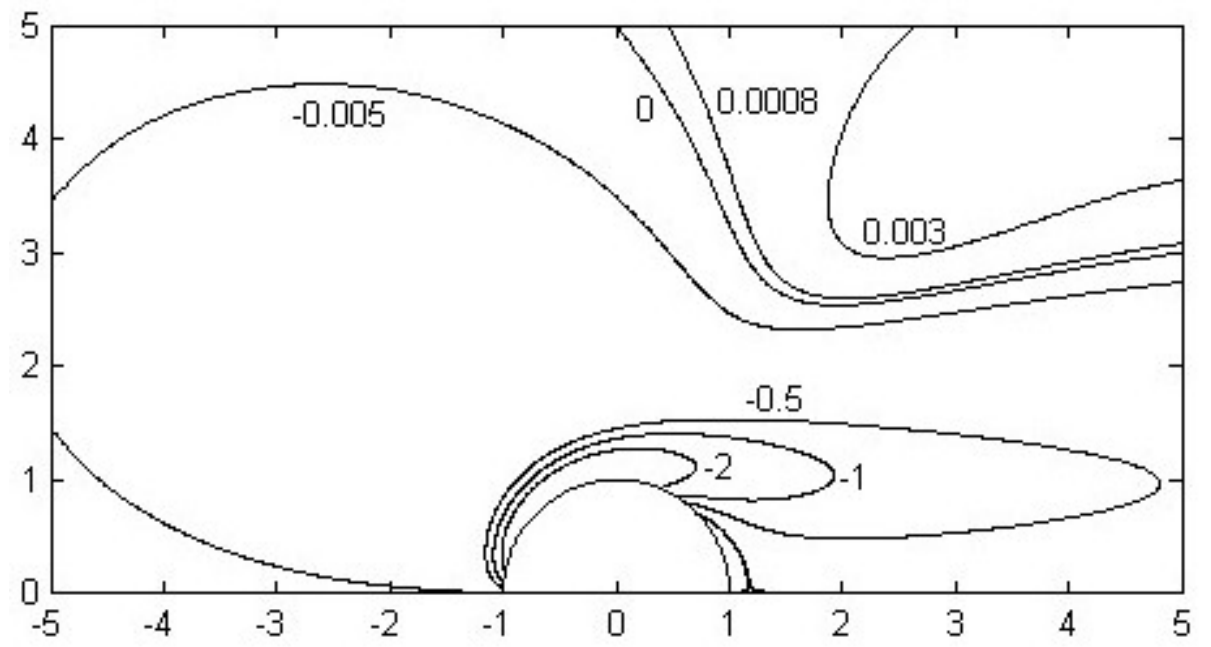

Figure 14. Vorticity lines for $R e=100$ and $M=10$.

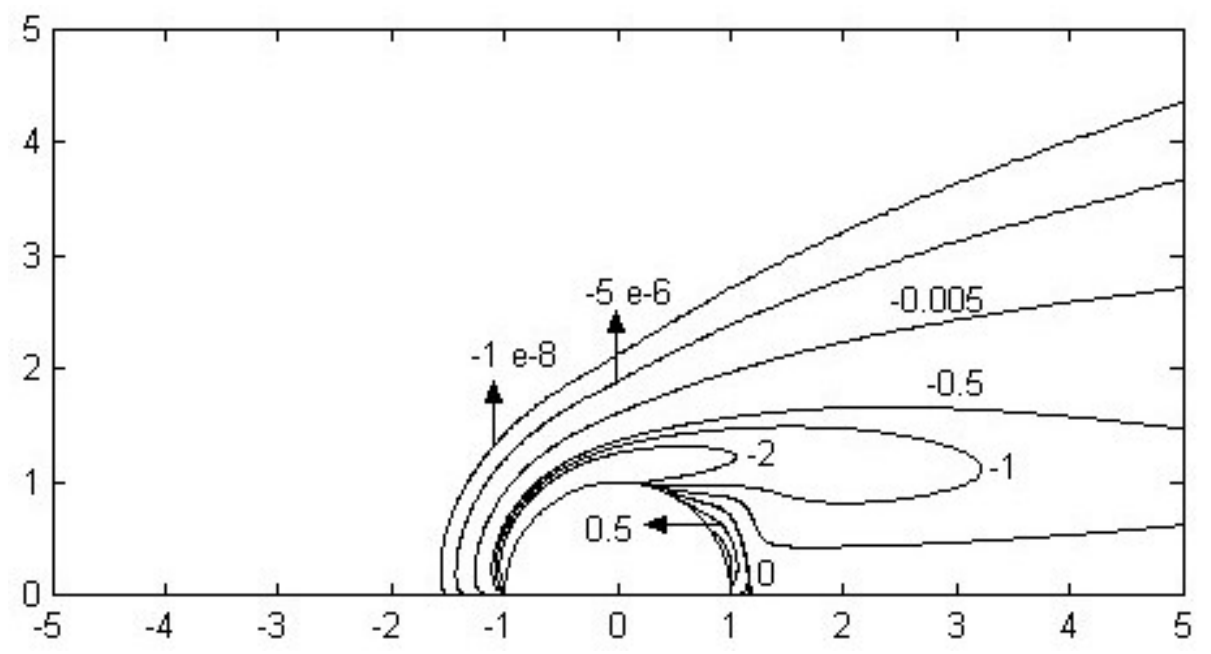

Figure 15. Vorticity lines for $\operatorname{Re}=200$ and $M=0$.

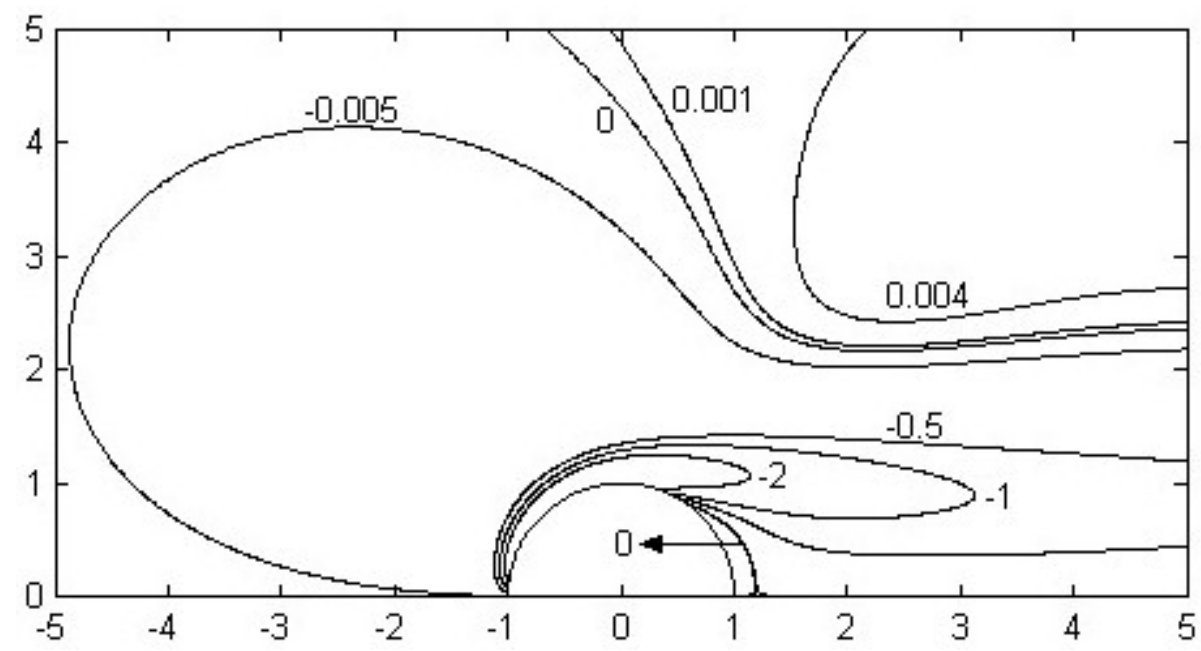

Figure 16. Vorticity lines for $R e=200$ and $M=14$. 


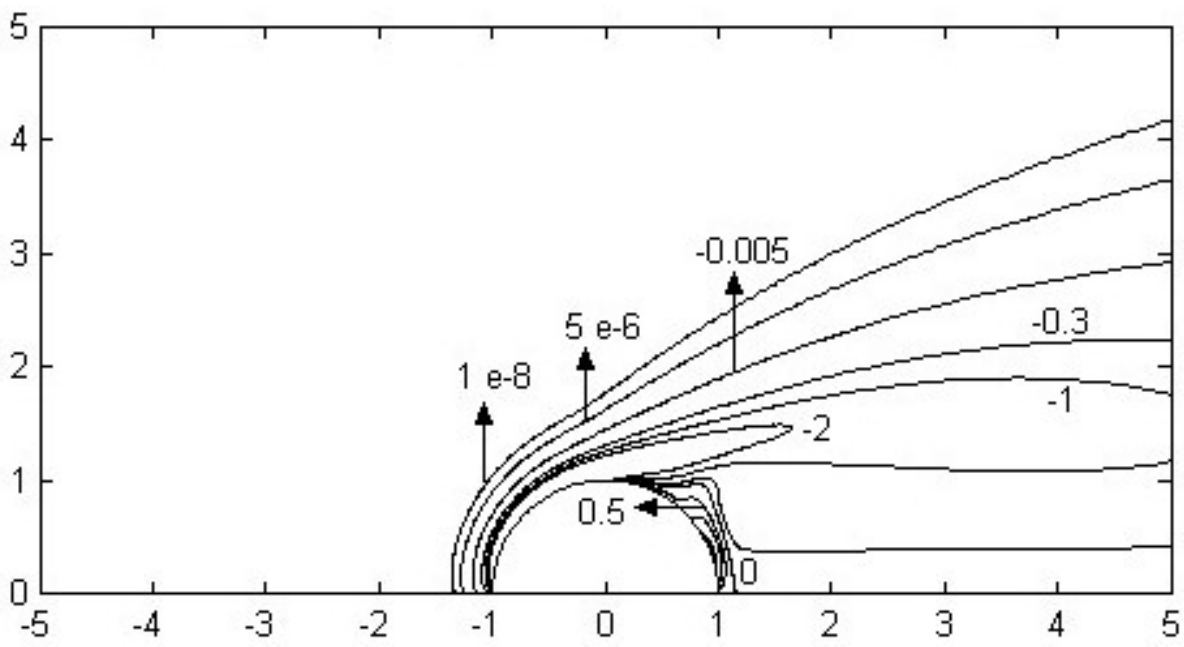

Figure 17. Vorticity lines for $R e=500$ and $M=0$.

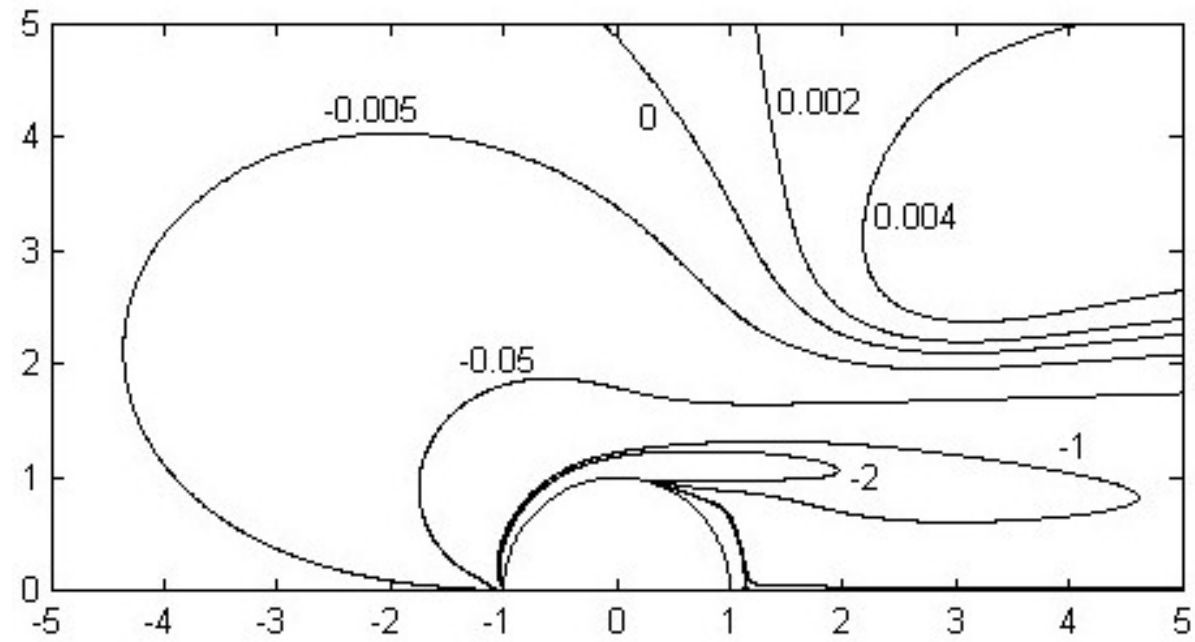

Figure 18. Vorticity lines for $R e=500$ and $M=20$.

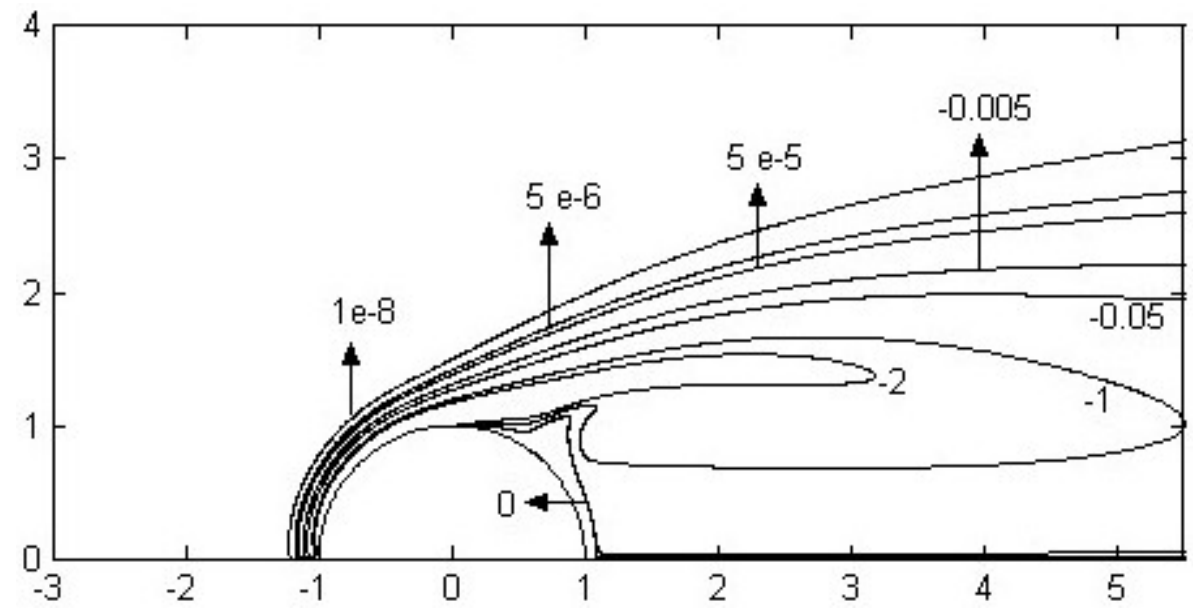

Figure 19. Vorticity lines for $R e=1000$ and $M=0$. 


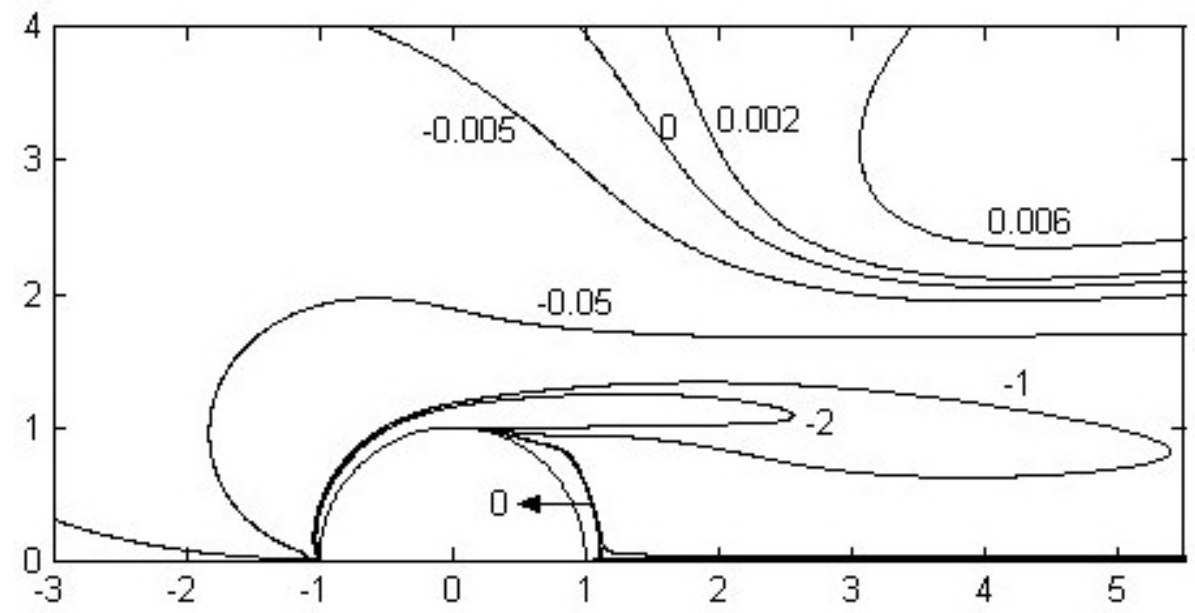

Figure 20. Vorticity lines for $R e=1000$ and $M=30$.

It can also be seen that the radial component (u) of fluid velocity near the sphere at $\theta=90^{\circ}$ is affected more compared to the transverse component $(\mathrm{v})$ as it $(\mathrm{u})$ is not parallel to the magnetic field (Figs. 21-22). As the Hartmann number increases, the thickness of the boundary layer adjoining the sphere surface decreases, indicating that it tends to zero for sufficiently large values of $M(M \gg 1)$ (Fig. 21). This is due to the enhanced velocity gradients required by the viscous stresses to compete with the large magnetic forces.

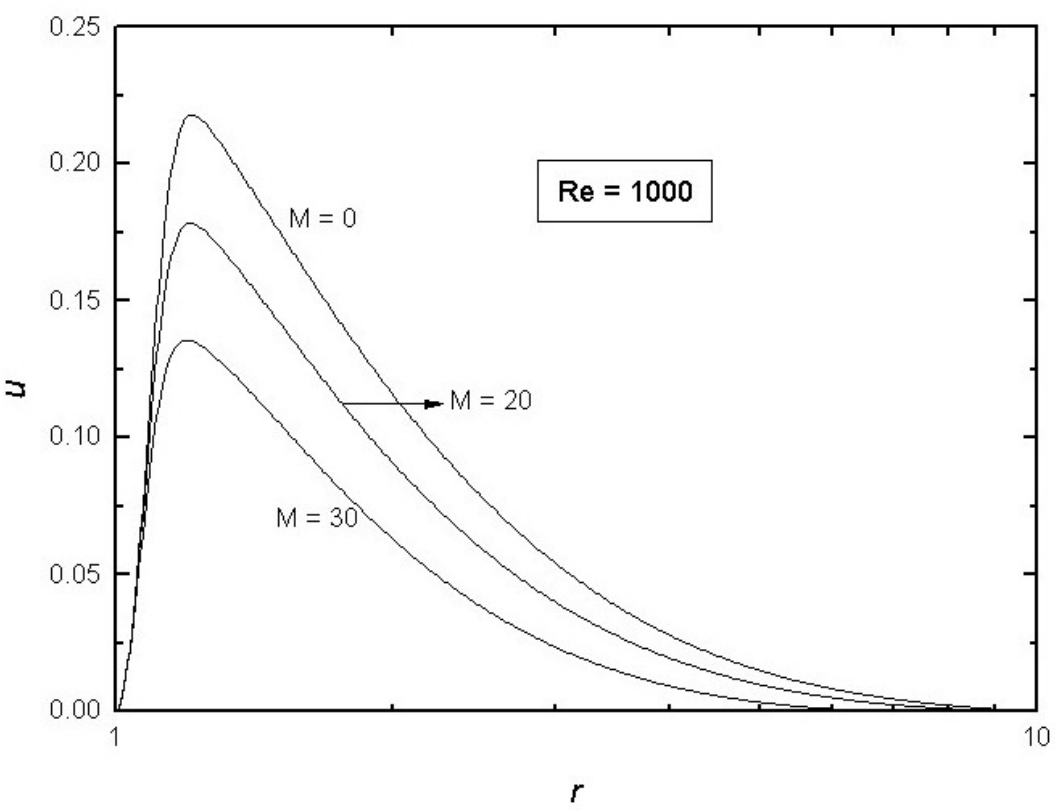

Figure 21. ' $r$ ' versus radial component of velocity at $\theta=90^{\circ}$ for $R e=1000$. 


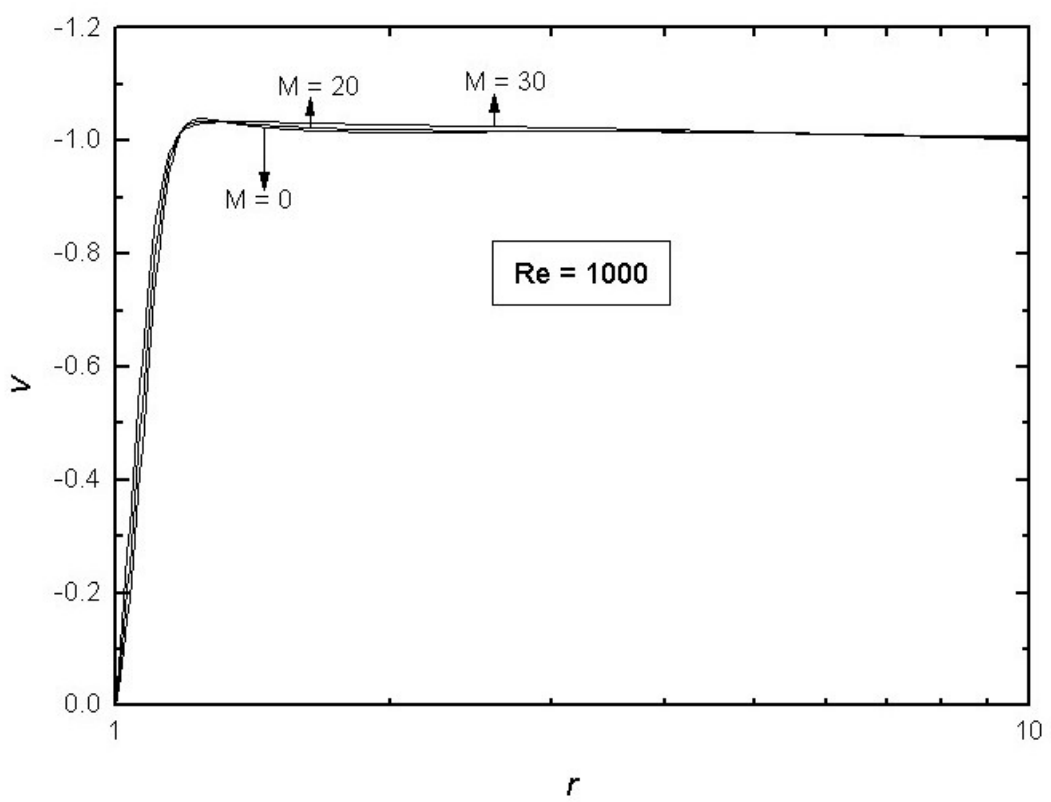

Figure 22. ' $r$ ' versus transverse component of velocity at $\theta=90^{\circ}$ for $R e=1000$.

The drag coefficients and surface pressure are calculated using the following relations:

Viscous drag coefficient

Pressure drag coefficient

$$
C_{v}=-\frac{4}{R e} \int_{0}^{\pi} \omega_{\xi=0} \sin ^{2} \theta d \theta
$$

$$
C_{p}=\frac{2}{\operatorname{Re}} \int_{0}^{\pi}\left\{\left(\omega+\frac{\partial \omega}{\partial \xi}\right)_{\xi=0} \sin ^{2} \theta\right\} d \theta
$$

Total drag coefficient

$$
C_{D}=C_{v}+C_{p}
$$

Surface pressure

$$
P_{\xi=0}(\theta)=1+\frac{8}{R e} \int_{0}^{\infty}\left(\frac{\partial \omega}{\partial \theta}\right)_{\theta=\pi} d \xi+\frac{4}{R e} \int_{\pi}^{\theta}\left(\frac{\partial \omega}{\partial \xi}+\omega\right)_{\xi=0} d \theta
$$

\begin{tabular}{|c|c|c|c|c|c|c|c|c|c|c|}
\hline \multirow[b]{2}{*}{$M$} & \multicolumn{3}{|c|}{$256 \times 256$} & \multicolumn{3}{|c|}{$512 \times 512$} & \multirow[b]{2}{*}{$\theta_{s}$} & \multirow[b]{2}{*}{$P_{\xi=o}(0)$} & \multirow[b]{2}{*}{$P_{\xi=0}(\pi)$} & \multirow{2}{*}{$(l / a)$} \\
\hline & $C_{v}$ & $C_{p}$ & $C_{D}$ & $C_{v}$ & $C_{p}$ & $C_{D}$ & & & & \\
\hline 0.00 & 0.28 & 0.25 & 0.53 & 0.28 & 0.25 & 0.53 & 53.33 & -0.08 & 1.10 & 2.80 \\
\hline 2.00 & 0.28 & 0.26 & 0.54 & 0.28 & 0.26 & 0.54 & 53.32 & -0.08 & 1.10 & 2.70 \\
\hline 5.00 & 0.28 & 0.27 & 0.55 & 0.28 & 0.27 & 0.55 & 50.88 & -0.08 & 1.10 & 2.50 \\
\hline 7.00 & 0.28 & 0.29 & 0.57 & 0.28 & 0.29 & 0.57 & 48.77 & -0.10 & 1.10 & 2.33 \\
\hline 9.00 & 0.28 & 0.32 & 0.60 & 0.28 & 0.32 & 0.60 & 46.32 & -0.12 & 1.10 & 2.19 \\
\hline 10.00 & 0.29 & 0.34 & 0.63 & 0.29 & 0.34 & 0.63 & 44.91 & -0.17 & 1.11 & 2.10 \\
\hline
\end{tabular}

The surface pressure values at $\theta=0^{\circ}$ and $\theta=180^{\circ}$, the wake length, the separation angle and the drag coefficient values in two different grids $256 \times 256$ and $512 \times 512$ are presented in Tables $1-4$.

Table 1. Drag coefficient values for $R e=100$ 
Table 2. Drag coefficient values for $R e=200$

\begin{tabular}{|c|c|c|c|c|c|c|c|c|c|c|}
\hline \multirow[b]{2}{*}{$M$} & \multicolumn{3}{|c|}{$256 \times 256$} & \multicolumn{3}{|c|}{$512 \times 512$} & \multirow[b]{2}{*}{$\theta_{s}$} & \multirow{2}{*}{$P_{\xi=o}(0)$} & \multirow{2}{*}{$P_{\xi=0}(\pi)$} & \multirow{2}{*}{$(l / a)$} \\
\hline & $C_{v}$ & $C_{p}$ & $C_{D}$ & $C_{v}$ & $C_{p}$ & $C_{D}$ & & & & \\
\hline 0.00 & 0.17 & 0.19 & 0.36 & 0.17 & 0.20 & 0.37 & 64.21 & -0.07 & 1.05 & 4.20 \\
\hline 5.00 & 0.17 & 0.20 & 0.37 & 0.17 & 0.20 & 0.37 & 62.80 & -0.08 & 1.05 & 3.80 \\
\hline 8.00 & 0.17 & 0.22 & 0.39 & 0.17 & 0.23 & 0.40 & 61.05 & -0.09 & 1.05 & 3.50 \\
\hline 10.00 & 0.17 & 0.24 & 0.41 & 0.17 & 0.25 & 0.42 & 59.30 & -0.10 & 1.05 & 3.30 \\
\hline 12.00 & 0.18 & 0.26 & 0.44 & 0.18 & 0.27 & 0.45 & 57.54 & -0.14 & 1.05 & 3.10 \\
\hline 14.00 & 0.18 & 0.28 & 0.46 & 0.18 & 0.29 & 0.47 & 55.44 & -0.16 & 1.06 & 2.90 \\
\hline
\end{tabular}

Table 3. Drag coefficient values for $R e=500$

\begin{tabular}{|c|c|c|c|c|c|c|c|c|c|c|}
\hline \multirow{2}{*}{$M$} & \multicolumn{4}{|c|}{$256 \times 256$} & \multicolumn{3}{|c|}{$512 \times 512$} & \multirow{2}{*}{$\theta_{s}(0)$} & \multirow{2}{*}{$P_{\xi=0}(\pi)$} & \multirow{2}{*}{$(l / a)$} \\
\cline { 2 - 12 } & $C_{v}$ & $C_{p}$ & $C_{D}$ & $C_{v}$ & $C_{p}$ & $C_{D}$ & & & & \\
\hline 0.00 & 0.09 & 0.14 & 0.23 & 0.09 & 0.14 & 0.23 & 78.60 & 0.12 & 1.02 & 5.50 \\
\hline 5.00 & 0.09 & 0.15 & 0.24 & 0.10 & 0.15 & 0.25 & 77.19 & 0.10 & 1.02 & 5.20 \\
\hline 10.00 & 0.10 & 0.16 & 0.26 & 0.10 & 0.16 & 0.26 & 74.74 & 0.06 & 1.02 & 4.90 \\
\hline 15.00 & 0.10 & 0.20 & 0.30 & 0.10 & 0.20 & 0.30 & 71.58 & -0.01 & 1.02 & 4.50 \\
\hline 20.00 & 0.11 & 0.23 & 0.34 & 0.11 & 0.23 & 0.34 & 68.42 & -0.06 & 1.02 & 4.25 \\
\hline
\end{tabular}

Table 4. Drag coefficient values for $R e=1000$

\begin{tabular}{|c|c|c|c|c|c|c|c|c|c|c|}
\hline \multirow[b]{2}{*}{$M$} & \multicolumn{3}{|c|}{$256 \times 256$} & \multicolumn{3}{|c|}{$512 \times 512$} & \multirow[b]{2}{*}{$\theta_{s}$} & \multirow[b]{2}{*}{$P_{\xi=o}(0)$} & \multirow[b]{2}{*}{$P_{\xi=0}(\pi)$} & \multirow{2}{*}{$(l / a)$} \\
\hline & $C_{v}$ & $C_{p}$ & $C_{D}$ & $C_{v}$ & $C_{p}$ & $C_{D}$ & & & & \\
\hline 0.00 & 0.05 & 0.09 & 0.14 & 0.05 & 0.10 & 0.15 & 85.45 & 0.15 & 1.01 & 6.00 \\
\hline 10.00 & 0.05 & 0.11 & 0.16 & 0.05 & 0.12 & 0.17 & 84.05 & 0.14 & 1.01 & 5.76 \\
\hline 20.00 & 0.05 & 0.14 & 0.19 & 0.05 & 0.15 & 0.20 & 80.35 & 0.10 & 1.01 & 5.51 \\
\hline 25.00 & 0.05 & 0.17 & 0.22 & 0.05 & 0.17 & 0.22 & 77.74 & 0.07 & 1.01 & 5.30 \\
\hline 30.00 & 0.06 & 0.19 & 0.25 & 0.06 & 0.19 & 0.25 & 75.64 & 0.04 & 1.01 & 5.12 \\
\hline
\end{tabular}

It can be seen from figures 23-25 that magnetic field tends to suppress the surface vorticity behind the sphere thereby competing with the viscous diffusion of vorticity out from the surface.

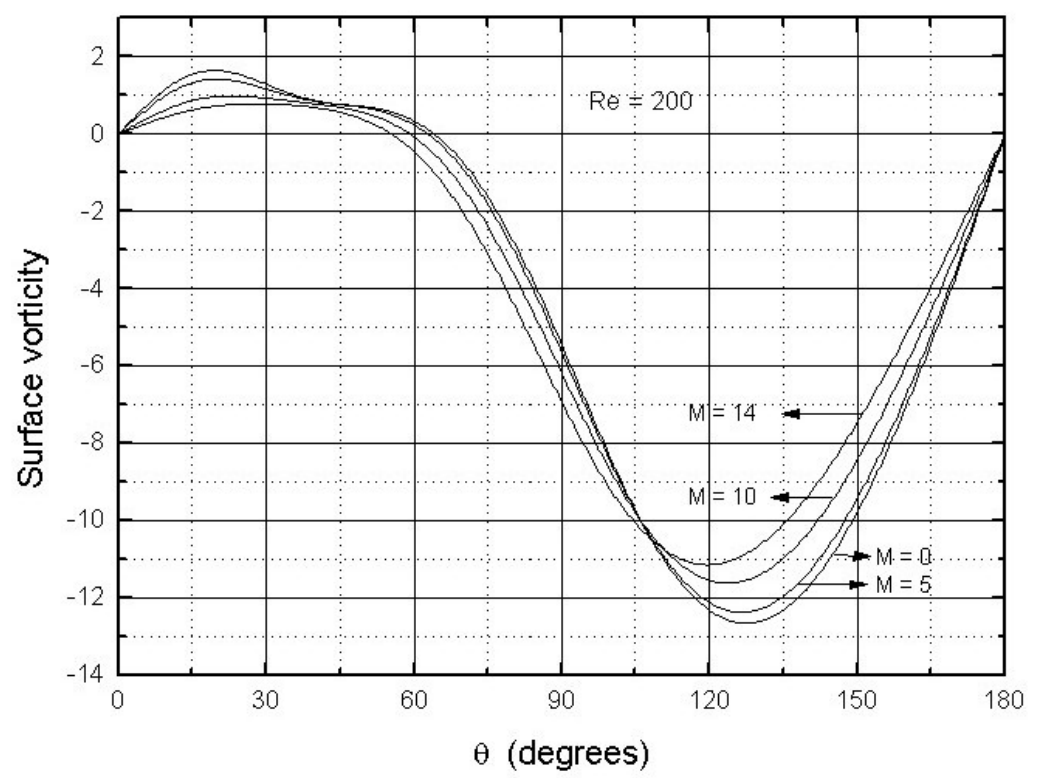

Figure 23. Theta versus surface vorticity for $R e=200$. 


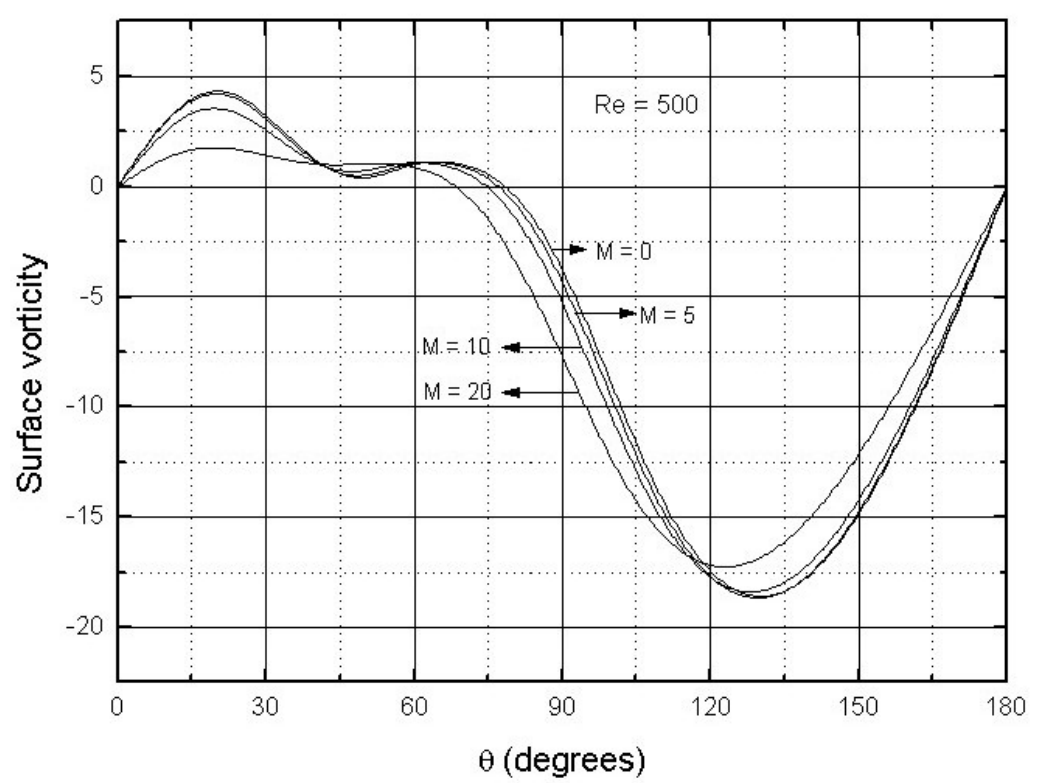

Figure 24. Theta versus surface vorticity for $R e=500$.

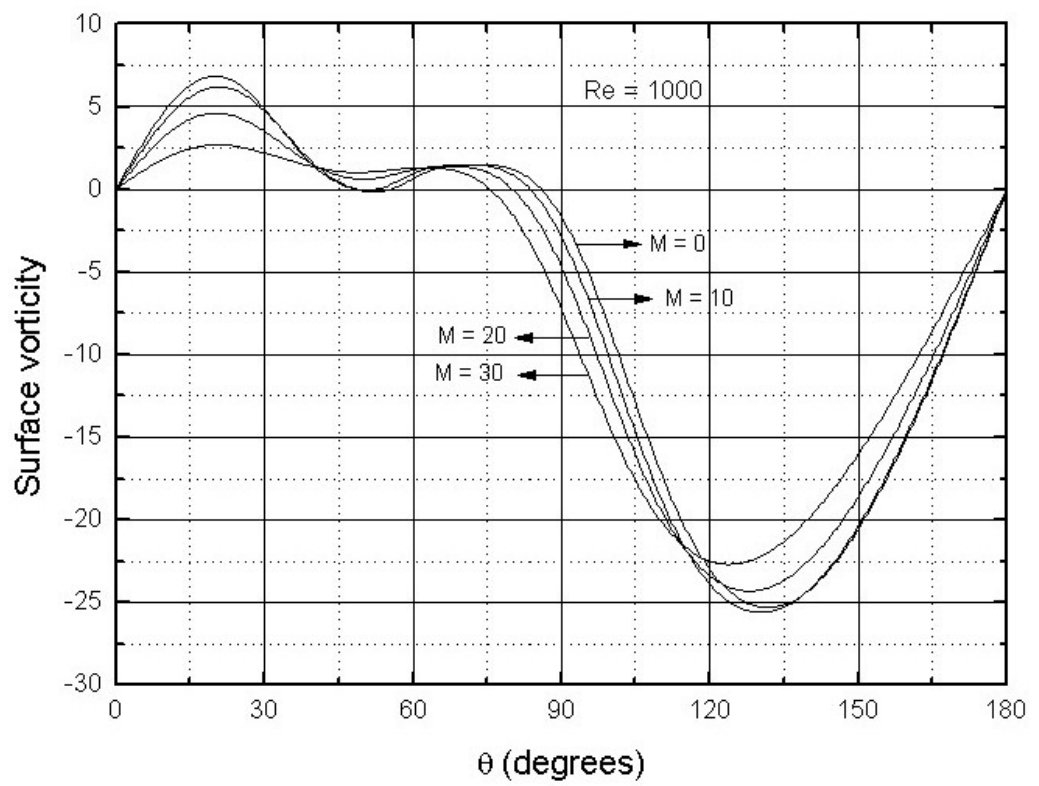

Figure 25. Theta versus surface vorticity for $R e=1000$.

We found that as the thickness of the boundary layer decreases, the increased velocity gradients at the surface will increase the pressure drop (Figs. 26-28) necessary to maintain the given flow rate. The observed flow field is in accordance with the assumption that the effect of magnetic field is the small perturbation of zero field potential flow. 


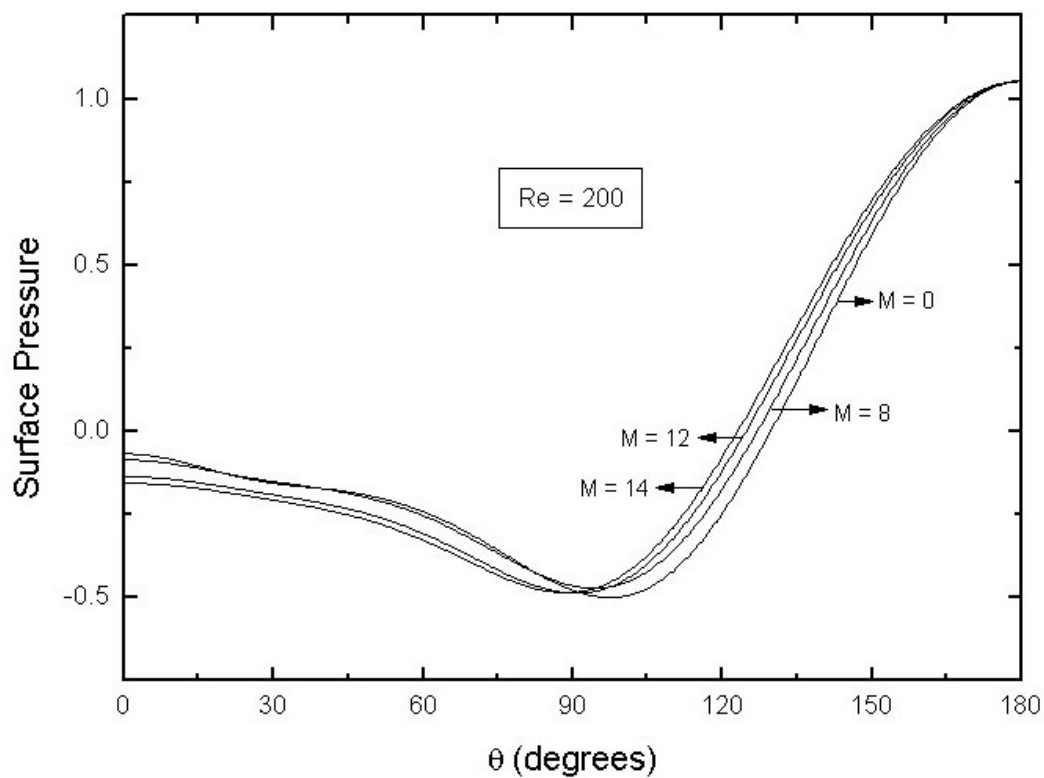

Figure 26. Theta versus surface pressure for $R e=200$.

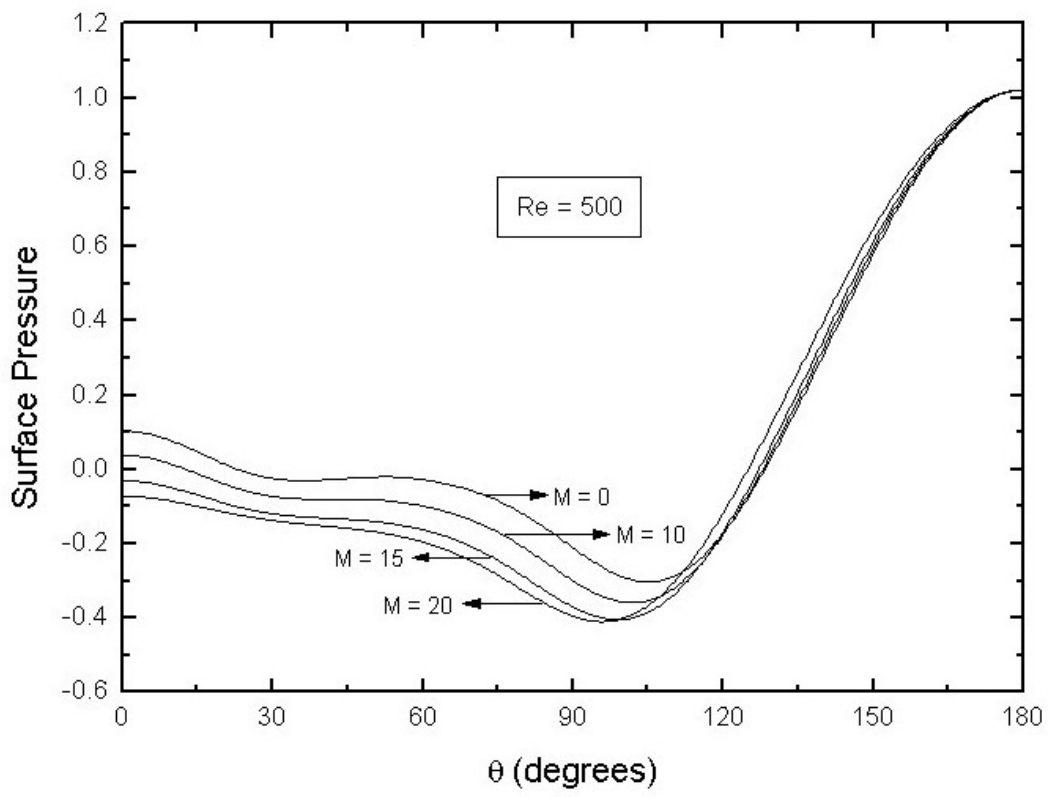

Figure 27. Theta versus surface pressure for $R e=500$. 


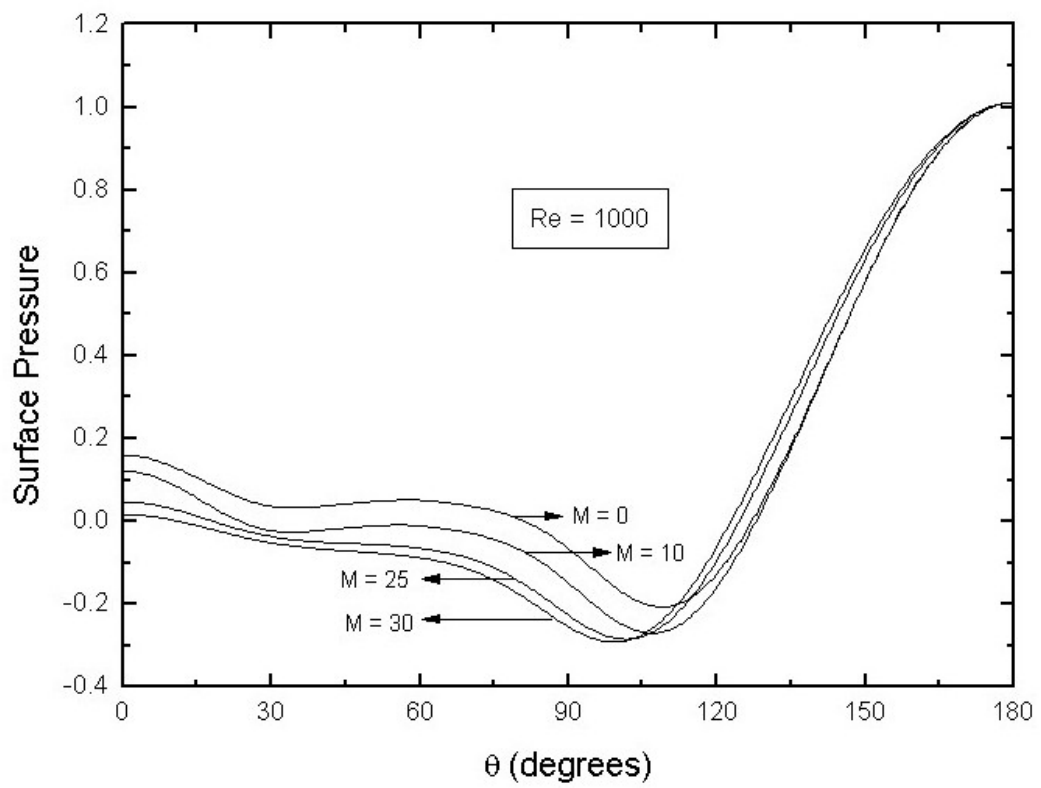

Figure 28. Theta versus surface pressure for $R e=1000$.

In the case of $M=0$, the drag coefficient values are in good agreement with the earlier work [Fornberg (1988), Juncu et al. (1990)]. The comparison of the drag coefficient values for $M=0$ is given in Table 5 and the graph of Reynolds number versus drag coefficient is presented in figure 29.

Table 5. Comparison of Drag coefficient values for $M=0$

\begin{tabular}{|c|c|c|c|c|}
\hline & \multicolumn{5}{|c|}{$R e$} \\
\hline References & 100 & 200 & 500 & 1000 \\
\hline Present results & 0.53 & 0.37 & 0.23 & 0.15 \\
\hline Fornberg (1988) & 0.54 & 0.38 & 0.24 & 0.16 \\
\hline Juncu et al.(1990) & 0.53 & - & - & - \\
\hline
\end{tabular}

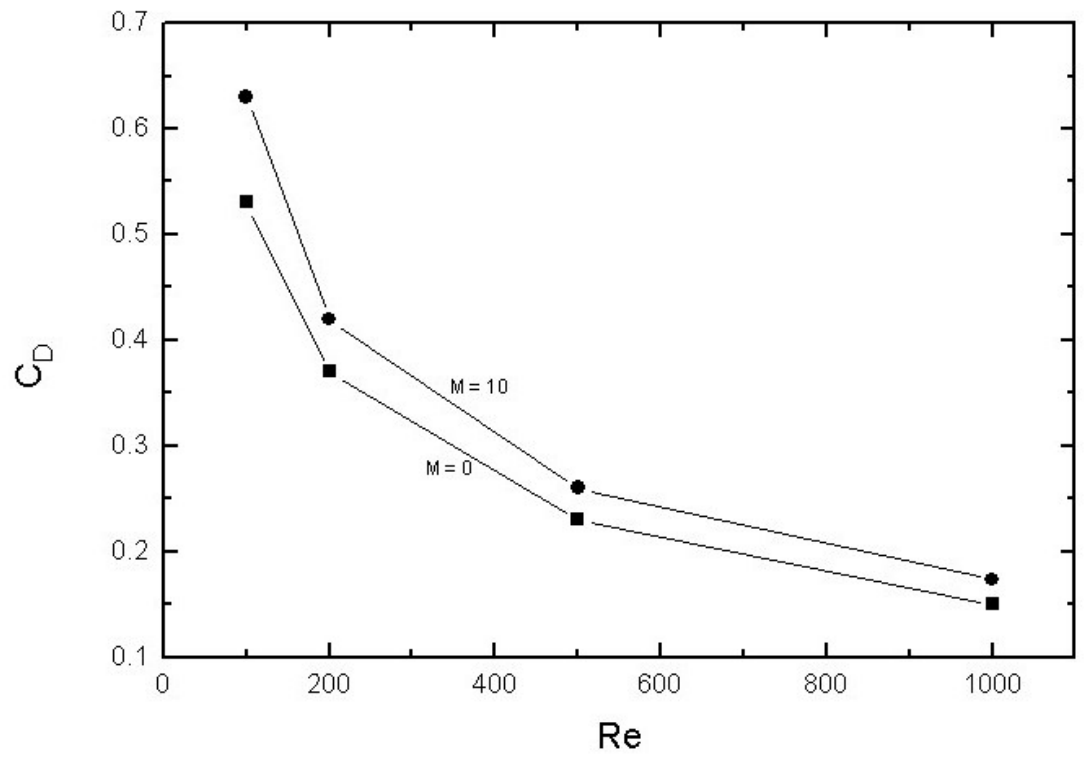

Figure 29. Reynolds number versus drag coefficient. 
Variations in the values of drag coefficient with Hartmann number are illustrated in figure 30.

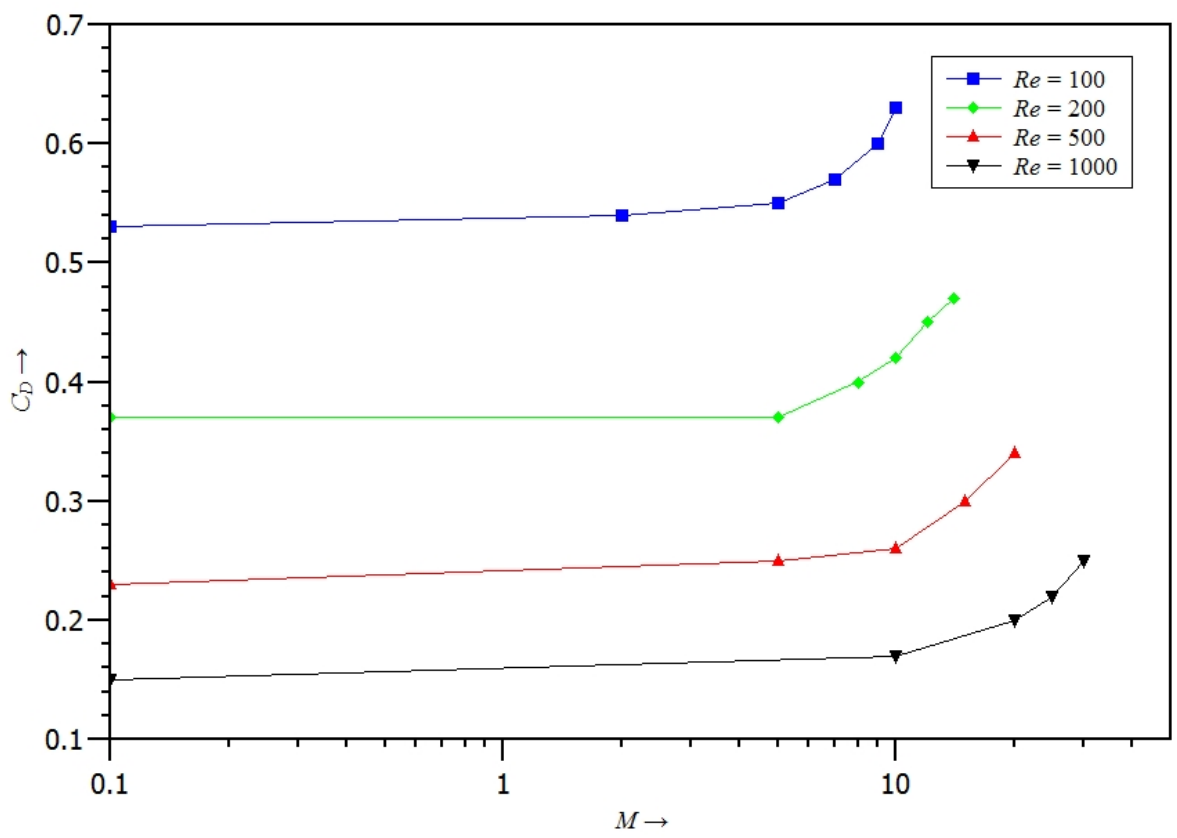

Figure 30. Variation of drag coefficient with Hartmann number

It can be noted that the flow pattern corresponding to some higher Reynolds number with magnetic field will be the same as the flow pattern corresponding to a lower Reynolds number with zero magnetic field. Hence we can infer that the application of an aligned magnetic field tends to stabilize the flow. We can also observe that the effect of magnetic field decreases as the Reynolds number is increased.

\section{Conclusions}

Steady flow of a finitely conducting fluid past a sphere at high Reynolds numbers is investigated using the multigrid method. Second order accurate solutions are obtained using the defect correction technique. A computational domain of size equal to sixty times the radius of the sphere is considered for the study. The effect of magnetic field on surface pressure, the wake length, the separation angle and the drag coefficient are studied and presented. It is found that the application of an aligned magnetic field tends to stabilize the flow by reducing the convection at higher Reynolds numbers. Also the magnetic field reduces the separation and slipping of the fluid at the surface of the sphere at the entire range of Reynolds numbers considered in this study. The application of magnetic field also tends to suppress the surface vorticity behind the sphere. It is further found that the drag coefficient increases with increasing magnetic field. The multigrid method with defect correction is found to give better results in terms of accuracy and computational cost as is evident from the fact that all the computations for the present study were carried out on a personal computer with core2duo processor $(2.2 \mathrm{GHz})$ and $2 \mathrm{GiB}$ RAM.

\section{References}

Cabannes H., 1970. Flow past a magnetised sphere (Theoretical Magnetofluid Dynamics), in Applied Mathematics and Mechanics, Vol. 13, p.175, Academic Press.

Chester W., 1957. The effect of a magnetic field on Stokes flow in a conducting fluid. Journal of Fluid Mechanics, Vol. 3, No. 3, pp.304-308.

Clift R., Grace J. R. and Weber M. E., 1978. Bubbles, Drops and Particles, Academic Press, New York.

Dennis S. C. R. and Walker J. D. A., 1971. Calculation of the steady flow past a sphere at low and moderate Reynolds numbers. Journal of Fluid Mechanics, Vol. 48, No. 4, pp.771-789.

Fornberg B., 1988. Steady viscous flow past a sphere at high Reynolds numbers. Journal of Fluid Mechanics, Vol. 190, pp.471489.

Garrett S.J. and Peake N., 2002. The stability and transition of the boundary layer on a rotating sphere. Journal of Fluid Mechanics, Vol. 456, pp.199-218.

Garrett S.J. and Peake N., 2004. The stability of the boundary layer on a sphere rotating in a uniform axial flow. European Journal 
of Mechanics, Vol. B 23, pp.241-253.

Garrett S.J. and Peake N., 2007. The absolute instability of the boundary layer on a rotating cone. European Journal of Mechanics, Vol. B 26, pp.344-353.

Garrett S.J., Hussain Z. and Stephen S.O., 2009. The cross-flow instability of the boundary layer on a rotating cone. Journal of Fluid Mechanics, Vol. 622, pp.209-232.

Juncu G. H. and Mihail R., 1990. Numerical solution of the steady incompressible Navier-Stokes equations for the flow past a sphere by a multigrid defect correction technique. International Journal for Numerical Methods in Fluids, Vol. 11, No. 4, pp.379-395.

Juncu G. H., 1999. A numerical study of steady viscous flow past a fluid sphere. International Journal of Heat and Fluid Flow, Vol. 20, No. 4, pp.414-421.

Kohama Y. and Kobayashi R., 1983. Boundary layer transition and the behavior of spiral vortices on rotating spheres. Journal of Fluid Mechanics, Vol. 137, pp.153-164.

Le Clair B. P., Hamielec A. E. and Pruppacher H. R., 1970. A numerical study of the drag on a sphere at low and intermediate Reynolds numbers. Journal of the Atmospheric Sciences, Vol. 27, No. 2, pp.308-315.

Leibovich S., 1967. Magnetohydrodynamic flow at a rear stagnation point. Journal of Fluid Mechanics, Vol. 29, No. 2, pp.401413.

Ludford G. S. S., 1960. The effect of an aligned magnetic field on oseen flow of a conducting fluid. Archive for Rational Mechanics and Analysis, Vol. 4, No.1, pp.405-411.

Raghava Rao C. V. and Sekhar T. V. S., 1993. Numerical solution of the slow translation of a sphere moving along the axis of a rotating viscous fluid. International Journal of Computational Fluid Dynamics, Vol. 1, No. 4, pp.351-359.

Raghava Rao C. V. and Sekhar T. V. S., 1995.Translation of a sphere in a rotating viscous fluid: A numerical study. International Journal for Numerical Methods in Fluids, Vol. 20, No. 11, pp.1253-1262.

Raghava Rao C. V. and Sekhar T. V. S., 2000. MHD Flow past a circular cylinder - a numerical study. Computational Mechanics, Vol. 26, No. 5, pp.430-436.

Savage M. D., 1972. Magnetohydrodynamic slow flow past a cylinder. International Journal of Engineering Science, Vol. 10, No. 2, pp.155-158.

Swarup S. and Sinha P. C., 1977. Magnetohydrodynamic flow past a circular cylinder. Journal of Applied Mathematics and Physics, Vol. 28, No. 1, pp.73-83.

Turkyilmazoglu M., 2006. Convective and absolute instabilities in the incompressible boundary layer on a rotating disk. Hacettepe Journal of Mathematics and Statistics, Vol. 35, pp.117-146.

Turkyilmazoglu M., 2011. Numerical and analytical solutions for the flow and heat transfer near the equator of an MHD boundary layer over a porous rotating sphere. International Journal of Thermal Sciences, Vol. 50, pp.831-842.

Wesseling P., 1991. Multigrid Methods in Fluid Dynamics, In Eds. W Hackbusch and U Trottenberg. Multigrid Methods-III, T337, Birkhäuser, Basel.

Wesseling P., 1980. Report NA-37. Delft University of Technology, The Netherlands.

Yosinobu H. and Kakutani T., 1959. Two-dimensional Stokes Flow of an electrically conducting fluid in a uniform magnetic field. Journal of the Physical Society of Japan, Vol. 14, No. 10, pp.1433-1444.

\section{Biographical notes}

Harish Kumar obtained his Ph. D. from Mangalore University, India and now working as a faculty in the Department of Physics, Pondicherry Engineering College, Pondicherry, INDIA. His special fields of interest are Computational and Theoretical Physics, and Optimization Techniques.

R. Rajathy obtained her B.E in Electrical and Electronics Engineering and M. E in power system with Distinction from Thiagarajar College of Engineering, Madurai. She received her Ph.D. degree from Pondicherry Central University. At present, she is working as a faculty in the Department of Electrical and Electronics Engineering, Pondicherry Engineering College, Pondicherry, India. Her special fields of interest are Computational techinques, Power System Optimization and Power System Restructuring.

Received August 2013

Accepted August 2013

Final acceptance in revised form September 2013 